\title{
BMSAP
}

Bulletins et mémoires de la Société d'Anthropologie de Paris

BMSAP

34 (1) | 2022

Entre vivants et morts : regards croisés sur une frontière relative et fluctuante

\section{Pratiques funéraires dans l'Arabie antique : la nécropole de Thaj}

Funerary practices in Ancient Arabia: the necropolis of Thaj

Marie Laguardia, Olivia Munoz, Jérôme Rohmer et Patrice Courtaud

\section{OpenEdition}

Journals

Édition électronique

URL : https://journals.openedition.org/bmsap/9483

DOI : $10.4000 /$ bmsap. 9483

ISSN : 1777-5469

Éditeur

Société d'Anthropologie de Paris

Référence électronique

Marie Laguardia, Olivia Munoz, Jérôme Rohmer et Patrice Courtaud, « Pratiques funéraires dans

I'Arabie antique : la nécropole de Thaj », Bulletins et mémoires de la Société d'Anthropologie de Paris [En ligne], 34 (1) | 2022, mis en ligne le 16 février 2022, consulté le 14 février 2023. URL : http:// journals.openedition.org/bmsap/9483; DOI : https://doi.org/10.4000/bmsap.9483

\section{cc) (i) $\Theta$}

Creative Commons - Attribution - Pas d'Utilisation Commerciale - Pas de Modification 4.0 International - CC BY-NC-ND 4.0

https://creativecommons.org/licenses/by-nc-nd/4.0/ 


\title{
Pratiques funéraires dans l'Arabie antique : la nécropole de Thaj
}

\author{
Funerary practices in Ancient Arabia: the necropolis of Thaj
}

\author{
Marie Laguardia ${ }^{1 *}$, Olivia Munoz ${ }^{2}$, Jérôme Rohmer ${ }^{3}$, Patrice Courtaud ${ }^{4}$ \\ 1. Université Paris 1 Panthéon-Sorbonne, UMR 7041 ArScAn, Paris, France \\ 2. CNRS, UMR 8215 Trajectoires, Paris, France \\ 3. CNRS, UMR 8167 Orient \& Méditerranée, Ivry-sur-Seine, France \\ 4. CNRS, UMR 5190 PACEA, Pessac, France \\ * laguardia.marie@gmail.com
}

Reçu : 3 août 2021 ; accepté : 20 décembre 2021 Bulletins et Mémoires de la Société d'Anthropologie de Paris

Cette note fait suite à une communication présentée lors des $1846^{\text {es }}$ journées de la Société d'Anthropologie de Paris dans le cadre de la session "Entre vivants et morts : regards croisés sur une frontière relative et fluctuante"

Résumé - En Arabie du Nord-Est, on observe un renouveau de l'occupation humaine à partir des IVe-III' siècles av. n. è., alors que la région devient un carrefour commercial entre l'Arabie du Sud, l'océan Indien, la Mésopotamie et le bassin méditerranéen. Cette "renaissance" se traduit notamment par l'apparition de vastes nécropoles tumulaires, qui semblent s'inscrire dans une tradition funéraire remontant au III ${ }^{\mathrm{e}}$ millénaire av. n. è. Le plus important des sites fondés à cette époque, Thaj, se trouve dans l'actuelle province orientale de l'Arabie Saoudite. Occupé jusqu'à la veille de l'Islam, le site se compose d'une ville fortifiée de 40 ha, de faubourgs et d'une vaste nécropole composée d'environ 1000 tombes. Depuis 2017, de nouveaux travaux de terrain (fouilles et prospections) ont été entrepris dans la nécropole de Thaj, dans le cadre d'une mission archéologique franco-saoudienne (CNRS/Saudi Heritage Commission). Combinés aux résultats des fouilles précédemment menées par le Département des Antiquités saoudien, ces travaux permettent désormais d'établir une typologie des monuments funéraires, de caractériser leurs techniques de construction et de mieux comprendre leur organisation spatiale au sein de la nécropole. Ils permettent également d'appréhender la diversité des sépultures implantées au sein de ces monuments, tant du point de vue de leur architecture que des traitements funéraires appliqués aux défunts. Enfin, une série de datations au radiocarbone effectuées sur les sépultures fouillées indique que certains monuments funéraires ont été utilisés sur plusieurs siècles, tandis que le matériel associé témoigne de la tenue de rituels funéraires, pratiqués au moment des funérailles et/ou lors de commémorations ultérieures.

Mots clés - Arabie orientale, époque préislamique, tumulus, traitement des défunts, rituels funéraires

\begin{abstract}
In north-eastern Arabia, a new phase of settlement expansion began in the $4^{\text {th }}-3^{\text {rd }}$ centuries BCE, as the region became a crossroads for trade between southern Arabia, the Indian Ocean, Mesopotamia and the Mediterranean. A remarkable aspect of this "renaissance" was the development of vast tumulus necropolises, which seems to have revived a regional building tradition dating back to the $3^{\text {rd }}$ millennium BCE. The largest settlement founded at this time, Thaj, is located in the eastern province of present-day Saudi Arabia and was occupied until shortly before the advent of Islam. The site consists of a walled city covering 40 ha, a suburban area and a large necropolis with at least 1000 tombs. In 2017, a new archaeological investigation of this necropolis was launched, as part of the Saudi-French Thaj Archaeological Project. The survey and the excavations carried out so far, together with the findings from previous fieldwork by the Saudi Department of Antiquities, have made it possible to establish a typology of the funerary monuments, characterize their construction techniques and better understand the spatial organization of the necropolis. The excavations have also revealed the diversity of the graves, which differ in their architecture and the funerary treatments applied to the dead. Finally, a series of radiocarbon dates obtained for the excavated graves shows that some of the funerary monuments were used and maintained for several centuries, while the associated material provides evidence for funerary rituals carried out either at the time of the funeral or during later commemorative ceremonies.
\end{abstract}

Keywords - Eastern Arabia, pre-Islamic period, burial mounds, treatment of the deceased, funerary rituals 


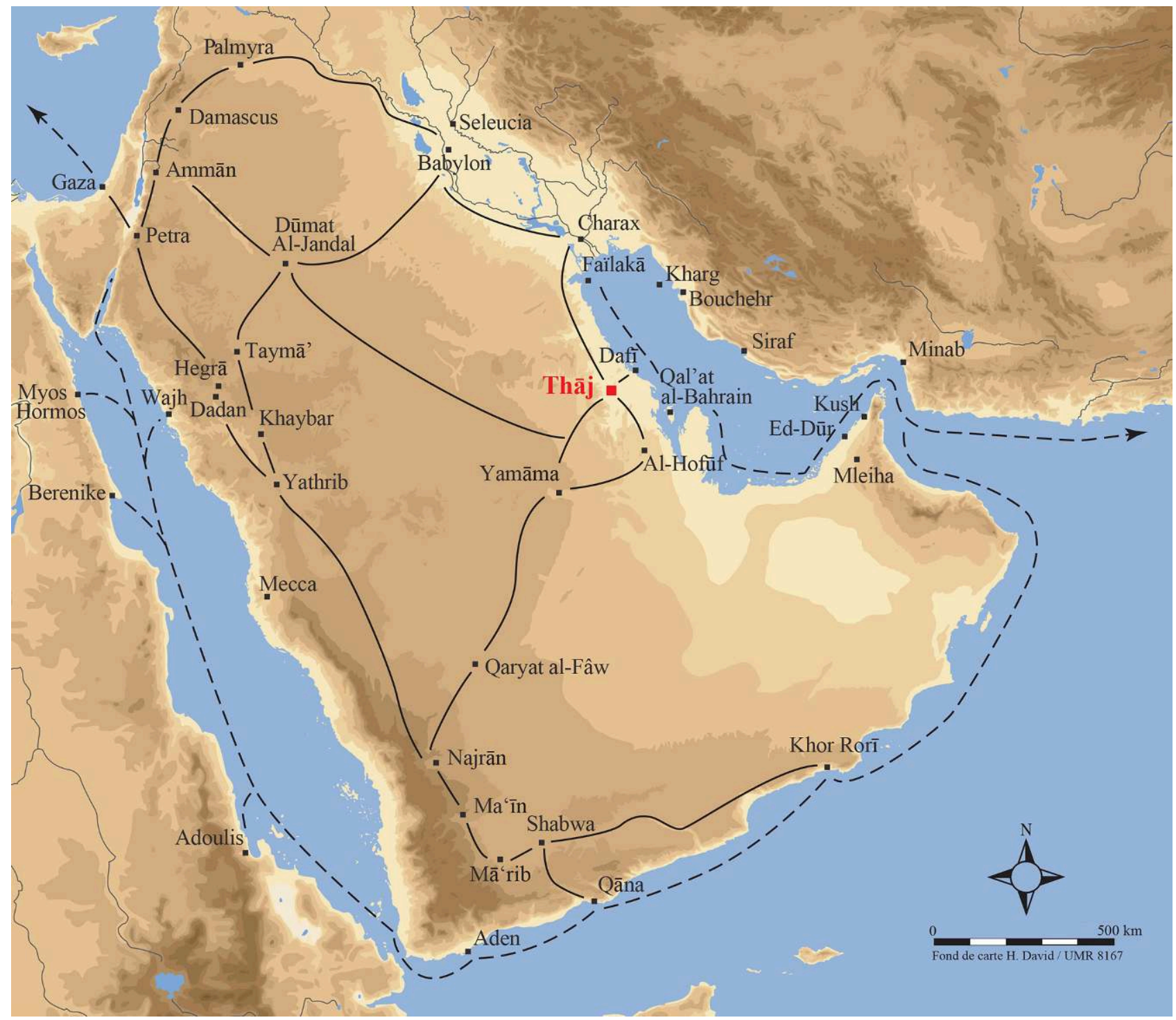

Figure 1. Carte des routes commerciales de l'Arabie antique et localisation de Thaj (DAO : J. Rohmer, fond de carte : H. David) / Map of trade routes in ancient Arabia and location of Thaj (CAD: J. Rohmer, map: H. David)

\section{Introduction}

À partir de l'époque hellénistique, et jusqu'à l'orée de l'époque sassanide (IVe-III ${ }^{e}$ siècles av.-III ${ }^{e}$ siècle de $n$. è.), l'Arabie du Nord-Est connaît une véritable "renaissance", marquée par un fort rebond de l'occupation humaine et par le développement d'une culture matérielle originale, à la faveur du développement du commerce à longue distance (Potts, 1990). Située au centre d'un réseau de routes terrestres et maritimes, la région connaît alors de nombreuses interactions culturelles avec le monde grec, la Mésopotamie et l'Arabie du Sud (figure 1).

Sur le plan funéraire, cette période se caractérise par le développement de grandes nécropoles tumulaires, qui s'insèrent dans une tradition remontant au $\mathrm{III}^{\mathrm{e}}$ millénaire av. n. è. Sur l'île de Bahreïn, au paysage déjà marqué par les grands champs de tumulus de la culture de Dilmun
(2200-1750 av. n. è. ; Højlund, 2007), on observe ainsi une nouvelle phase de formation de tumulus lors de la période dite "Tylos" (300 av.-400 de n. è. ; Salman et Andersen, 2009). Sur le continent, la vaste nécropole tumulaire de Dhahran, en usage depuis la fin du III millénaire, connaît des réoccupations aux époques hellénistique, parthe et sassanide (300 av.-600 de n. è. ; Zarins et al., 1984), tandis que de nouveaux ensembles tumulaires se développent sans doute dès l'époque hellénistique au Jabal Kenzan (Potts, 1990:57), à Ayn Jawan (Bowen et al., 1950 ; Potts, 1990 : 204-208 ; 1993) et surtout à Thaj (Bibby, 1973 ; al-Hashash et al., $2001 ; 2002 ; 2005 ; 2006)^{1}$.

1 Il semble que la situation soit différente sur l'île de Tarut, où l'ensemble des sépultures hellénistiques identifiées provient d'un unique tertre, de forme oblongue (Potts, $1990: 51-53$ ). 
Cependant, à la différence des nécropoles de Bahreïn/ Tylos, désormais bien connues grâce aux fouilles des vingt dernières années, les nécropoles du continent n'ont fait l'objet jusqu'à récemment que de prospections rapides et de fouilles ponctuelles, de surcroît sans véritable étude anthropologique. Ce manque de données est d'autant plus dommageable que l'on ne saurait purement et simplement généraliser à l'ensemble de l'Arabie du Nord-Est les observations faites dans le contexte insulaire et particulièrement cosmopolite de la Bahreïn antique ${ }^{2}$. Les fouilles entreprises depuis 2016 sur le site de Thaj, dans le cadre d'une mission franco-saoudienne copilotée par le CNRS et le Ministère de la Culture d'Arabie Saoudite, constituent donc une occasion privilégiée de combler cette lacune et de caractériser les pratiques funéraires de l'Arabie du Nord-Est, en lien avec la fouille d'un grand établissement urbain ${ }^{3}$.

Situé à environ $90 \mathrm{~km}$ de la côte du Golfe, Thaj est le plus grand site archéologique préislamique connu en Arabie orientale. Occupée entre les IVe-III ${ }^{\mathrm{e}}$ siècles av. n. è. et le $\mathrm{IV}^{\mathrm{e}}$ siècle de n. è., voire jusqu'aux $\mathrm{VI}^{\mathrm{e}}-\mathrm{VII}^{\mathrm{e}}$ siècles de n. è. dans certaines zones, la ville antique couvre à elle seule plus de 50 ha (Rohmer, 2019). Certains proposent de l'identifier à l'ancienne Gerrha, riche cité commerçante d'Arabie orientale mentionnée par les sources grecques et latines, bien que cette identification ne fasse pas consensus (voir entre autres Potts, 1990 : 85-97 ; Robin, Prioletta, 2013 ; Robin, 2016). La nécropole du site compte plus d'un millier de monuments funéraires, dont seulement six avaient fait l'objet de fouilles ou de sondages avant notre intervention, dans le cadre de la prospection danoise de G. Bibby en 1968 (1973) puis de fouilles de sauvetage du Département des antiquités saoudien, entre 1998 et 2001 (al-Hashash et al., 2001 ; 2002 ; al-Hashash 2005 ; 2006).

Au sein de la mission franco-saoudienne de Thaj, un programme d'archéologie funéraire a été lancé en 2017, en partenariat avec l'Institut des Déserts et des steppes, dans le but d'étudier la nécropole du site dans une perspective globale, depuis son organisation d'ensemble jusqu'aux traitements et aux rituels funéraires. Après trois campagnes de terrain (2017-2019), de nouvelles hypothèses peuvent être formulées concernant le paysage funéraire, les traitements funéraires et le maintien du lien entre les vivants et les morts.

2 Une différence frappante entre les tombes de Bahreïn (et celles de l'île de Tarut) et celles du continent réside dans le type des stèles funéraires. Alors que l'on trouve à Bahreïn des stèles anthropomorphes ou figuratives inspirées du monde mésopotamien et levantin, parfois associées à une courte formule grecque, les stèles trouvées sur le continent semblent comporter exclusivement des inscriptions sémitiques écrites dans la langue et la graphie locale, inspirée de l'alphabet sud-arabique.

3 Mission menée par l'UMR 8167 du CNRS et le Ministère de la Culture d'Arabie Saoudite, avec le soutien du Ministère de l'Europe et des affaires étrangères, de Torathuna (fonds RSE de l'entreprise Satorp) et d'Éveha International.

\section{Thaj : un paysage marqué par les tombes tumulaires}

Installé au sud d'une sebkha (dépression saline inondée en hiver), le site de Thaj se compose d'une ville fortifiée de 40 ha, de faubourgs d'environ 10 ha et d'une vaste nécropole comportant plus de 1000 structures funéraires monumentales (Laguardia et al., 2019).

\section{Typologie et modalités de construction des monuments funéraires}

La nécropole s'étend au nord-ouest, nord-est et principalement au sud de la ville antique (figure 2). Lors des premières explorations du site (Mandaville, 1963 ; Bibby, 1973), deux principaux types de monuments funéraires ont été identifiés à Thaj : les tumulus et les cercles blancs. Les premiers correspondent à des monticules, plus ou moins coniques, formés par une accumulation artificielle de terre (figure 3 ). Les seconds sont des cercles dont le périmètre est matérialisé au sol par des fragments de calcaire blanc, particulièrement visibles sur les photographies aériennes (figure 4). Ces cercles sont parfois surélevés, ce qui crée une cuvette dans la partie centrale. Nous avons également identifié une autre forme de sépulture, des cairns, correspondant à de petits amas de pierres, ainsi que des cistes isolées.

Sur la base d'un examen des photographies aériennes et de prospections pédestres systématiques, 787 tumulus, 219 cercle blancs, 32 cairns et 13 cistes isolées ont pu être inventoriés et enregistrés. Si ce premier inventaire a permis de dénombrer et de distinguer les principaux types de tombes, les nouvelles données de terrain incitent aujourd'hui à nuancer la distinction entre "tumulus" et "cercle blanc". En effet, les observations et les fouilles récentes ont révélé une multitude de cas intermédiaires qui suggèrent que ces deux catégories correspondent en réalité à des stades de construction ou à des degrés d'élaboration différents d'un seul et même type de monument funéraire.

On observe systématiquement, dans les tumulus qui ont été fouillés, la présence d'un cercle blanc à la base du tertre. D'autre part, il semble que les pentes des tumulus aient été elles aussi recouvertes de fragments de calcaire blanc.

Ainsi, il est possible de restituer différentes étapes de la construction des monuments : la première semble être la réalisation d'une enceinte circulaire grâce à un dépôt de fragments calcaires blancs provenant de résidus de taille (tumulus 71), au sein de laquelle une ou plusieurs sépultures individuelles peuvent être implantées. Le tumulus 340 a livré une enceinte circulaire constituée d'un muret de pierres calcaires blanches, conservé par endroits sur trois assises. Ce cas particulier, couplé aux données de prospection, suggère que dans certains cas les cercles blancs pouvaient également être formés par des murs construits qui se seraient délités au cours du temps. Dans un second temps, trois cas de figure sont possibles. Dans le premier, l'ensemble de l'espace funéraire est directement recouvert par un tertre unique, composé de sable et de pierres calcaires. Dans le 


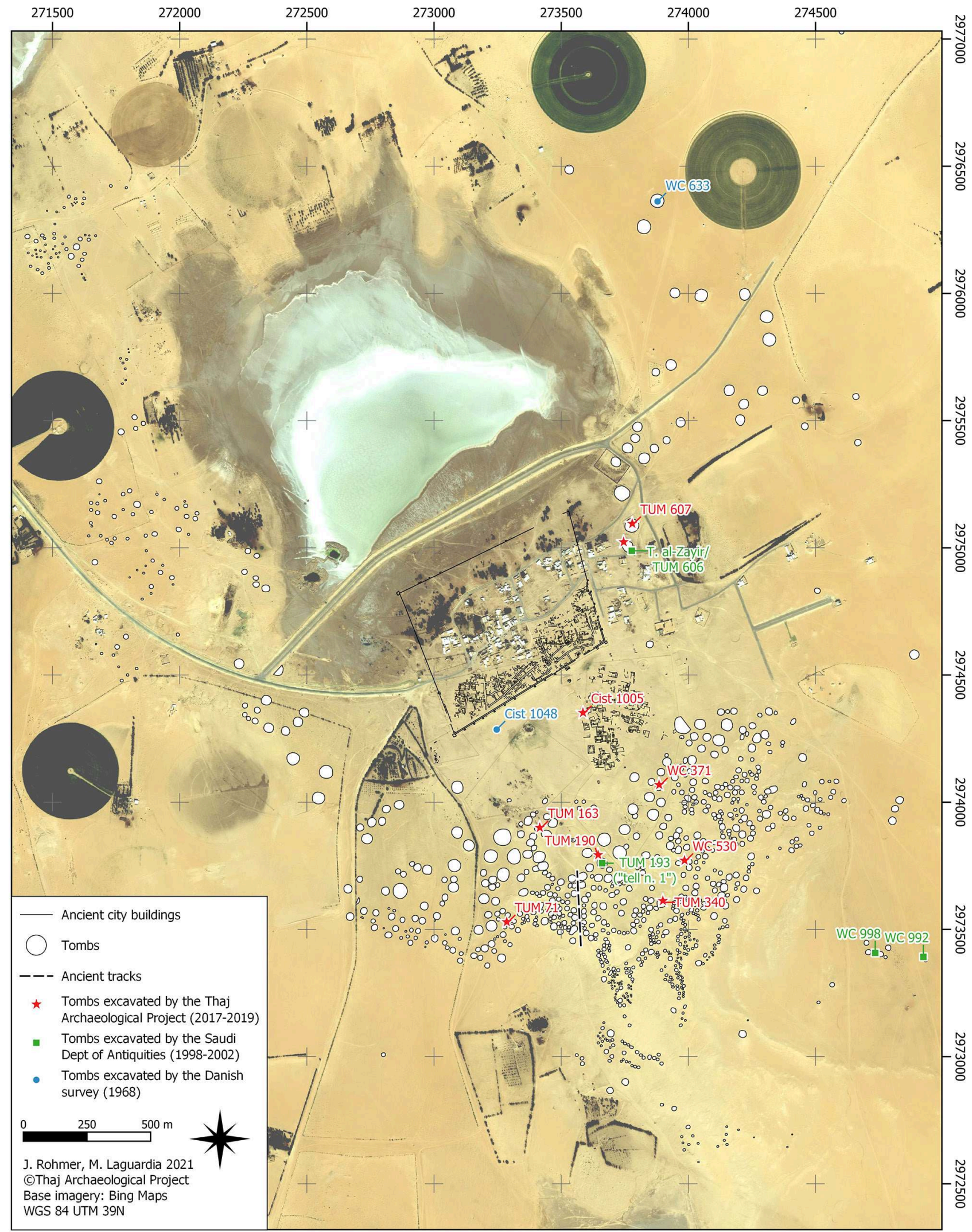

Figure 2. Plan de Thaj où figure la localisation des tombes fouillées (Thaj Archaeological Project. J. Rohmer, M. Laguardia 2021) / Map of Thaj showing the location of the excavated tombs (Thaj Archaeological Project. J. Rohmer, M. Laguardia 2021) 


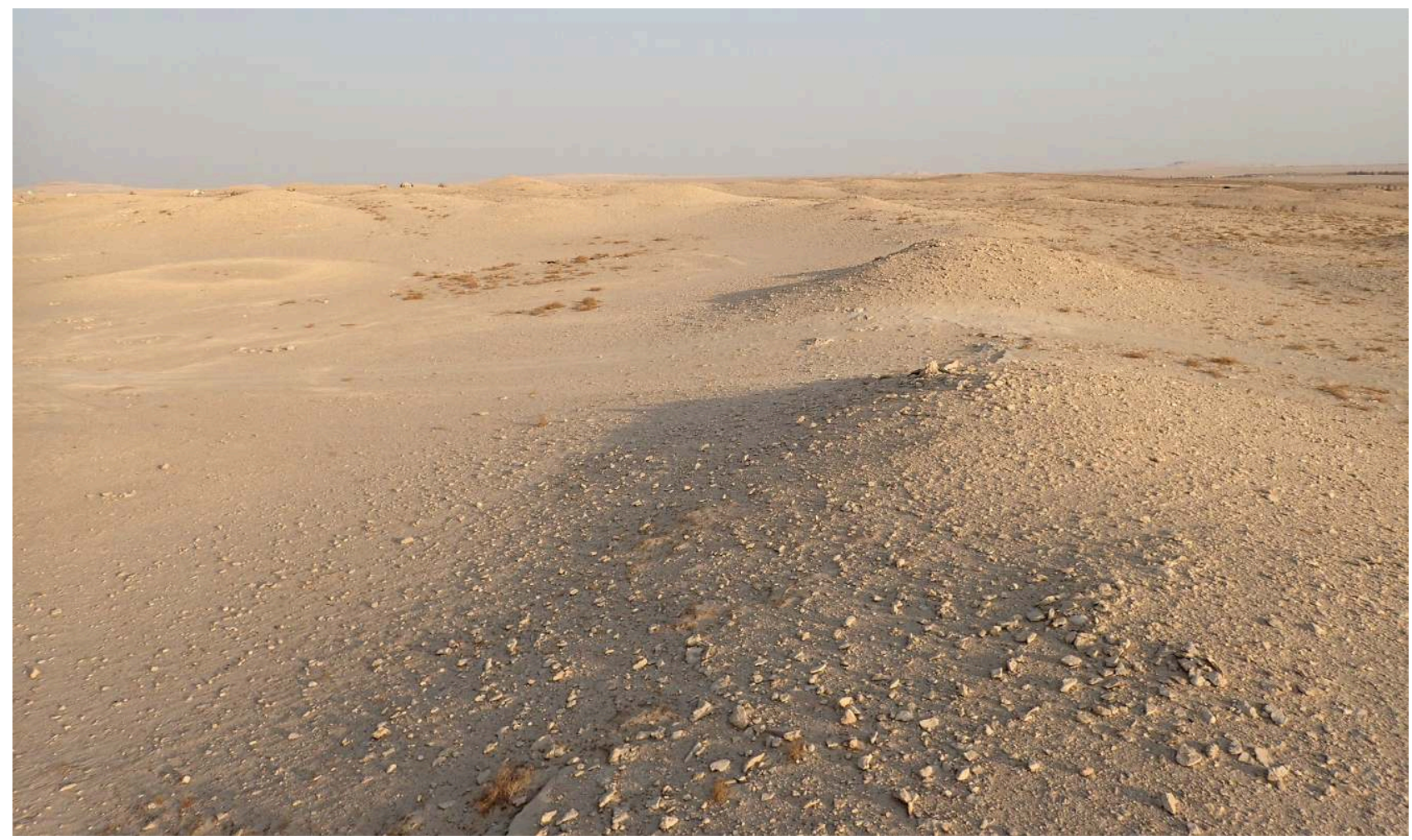

Figure 3. Tumulus de la nécropole sud (Thaj Archaeological Project. M. Laguardia 2021) /

Tumulus of the southern necropolis (Thaj Archaeological Project. M. Laguardia 2021)

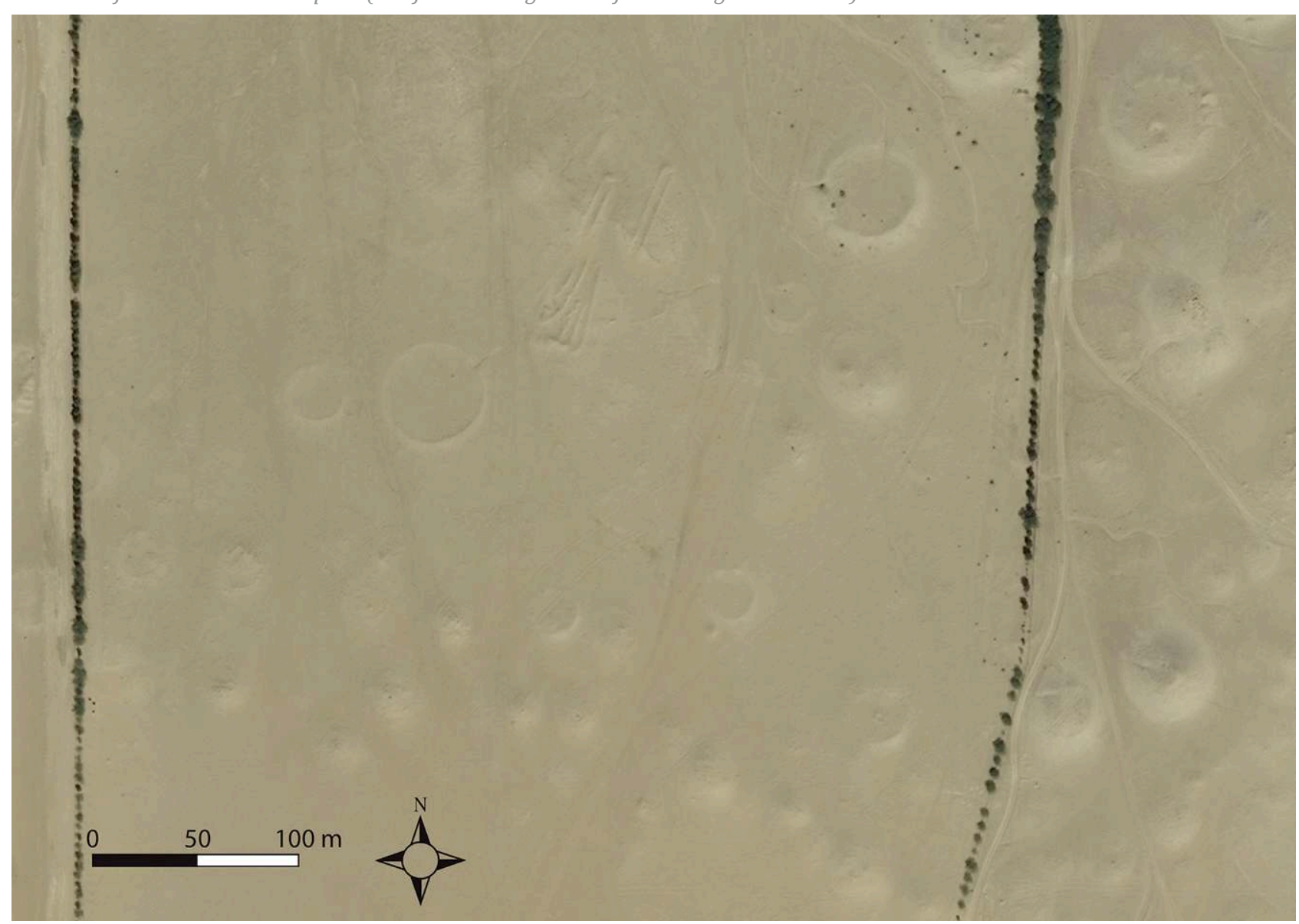

Figure 4. Photographie aérienne des monuments funéraires de Thaj (Thaj Archaeological Project. M. Laguardia 2021) / Aerial photograph of the funerary monuments of Thaj (Thaj Archaeological Project. M. Laguardia 2021) 
second cas (tumulus 163), les sépultures individuelles sont chacune surmontées d'un monticule. Ces monticules s'agglomèrent au cours du temps et finissent par être rassemblés sous un grand tumulus : c'est ce processus qui a été observé pour la formation des tumulus de la période Tylos à Bahreïn (Andersen et Salman, 2006). Pour finir, la surface des tumulus est souvent revêtue de fragments calcaires blancs. Les fouilles ont révélé des phases d'entretien et de réaménagement des monuments funéraires au cours du temps, notamment par l'adjonction réitérée de fragments calcaires blancs, visible en stratigraphie dans les tranchées de sondage (cercle blanc 371).

La raison pour laquelle certains enclos sont restés à l'état de cercle blanc, sans être scellés par un tertre, demeure pour le moment inconnue. Il semble que ces grands cercles aient formé des enclos funéraires permettant d'accueillir de nouvelles tombes sur plusieurs générations. La construction d'un tertre scellait le monument funéraire et impliquait probablement de renoncer à de nouvelles inhumations. Faut-il imaginer que les cercles blancs ne soient que des tumulus laissés ouverts en prévision de futurs décès ? Ou faut-il au contraire considérer qu'il s'agit d'un choix délibéré, et qu'en dépit de leur parenté constructive avec les tumulus, les cercles blancs constituaient malgré tout un type de monument funéraire particulier aux yeux des habitants de Thaj ?

Il faut signaler, pour finir, un cas particulier. Au nordest de ville antique, la fouille d'un tumulus monumental de $45 \mathrm{~m}$ de diamètre (Tall al-Zayir) par une équipe du Département des Antiquités saoudien (al-Hashash et al., 2001 ; 2002) a mis au jour une unique sépulture contenant un riche mobilier funéraire daté des $\mathrm{I}^{\mathrm{er}}-\mathrm{II}^{\mathrm{e}}$ siècles de $\mathrm{n}$. è. La ciste était installée au sein d'une accumulation de terre, de cendre, de tessons de céramique et d'os animaux. En 2018, la reprise de la fouille par la mission franco-saoudienne a révélé que ce monticule constituait à l'origine un grand dépotoir, dont la phase initiale correspond aux déchets d'un four de potier antérieur de plusieurs siècles à la sépulture (IV -III $^{\mathrm{e}}$ siècles av. n. è.). Dans ce cas, il semble donc qu'un monticule préexistant ait été utilisé pour installer une sépulture unique. La fouille d'un autre grand tumulus situé dans la même zone (malheureusement interrompue par la découverte d'un cimetière islamique) a mis au jour le même type de dépôts. Au total, on compte jusqu'à sept tumulus monumentaux de ce type dans la zone située au nord-est de la ville antique.

Quel que soit leur type, les tombes monumentales de Thaj ont profondément modifié le paysage par leurs dimensions et par leur habillage de fragments de calcaire blanc, qui accentue leur visibilité. Cette volonté de marquer l'espace funéraire se manifeste également par l'emplacement des tombes dans la nécropole.

\section{Analyse spatiale de la distribution des tombes}

Les tombes de Thaj, tumulus et cercle blanc confondus, mesurent entre 3 et $68 \mathrm{~m}$ de diamètre (tableau 1 ). On remarque que les petites structures (3-15 $\mathrm{m}$ de diamètre) sont majoritaires dans la nécropole puisqu'elles représentent presque la moitié des tombes (46\%).

\begin{tabular}{|l|c|c|c|c|}
\hline Diamètre (m) & $3-15$ & $15-25$ & $25-40$ & $40-68$ \\
\hline Tombes & $46 \%$ & $34 \%$ & $15 \%$ & $5 \%$ \\
\hline
\end{tabular}

Tableau 1. Nombre de structures tumulaires en fonction des catégories de taille (discrétisation selon l'algorithme de Jenks) / Number of tumulus structures by size categories (discretization according to the Jenks algorithm)

Ces résultats ont été intégrés au SIG de la nécropole et peuvent être présentés sous la forme d'une carte de distribution selon leurs dimensions (figure 5). Cette dernière permet de voir que les monuments funéraires les plus imposants sont situés au nord-est et à l'ouest de la ville, tandis que les plus petits se développent au nord-ouest. Au sud, les deux catégories de taille sont présentes mais on remarque que les tombes les plus monumentales sont proches de la ville antique alors que les petites sont plus éloignées. Les tombes se répartissent autour de la zone urbaine avec un réel souci de visibilité, caractérisé d'une part par la monumentalité des grandes tombes aux abords de la ville et, d'autre part, par l'implantation des petites structures sur des points hauts. En effet, les petits monuments funéraires suivent la topographie naturelle de la zone en se positionnant sur des éminences calcaires et des lignes de crêtes, qui accentuent leur visibilité. Ce phénomène est perceptible au sud de la nécropole.

Bien que l'étude céramique du matériel de prospection soit encore en cours, il semble que la distance des tombeaux par rapport à la ville et leur taille ne soient pas fonction de leur chronologie. Alors que le matériel de prospection indique une période d'utilisation de la nécropole allant des $\mathrm{IV}^{\mathrm{e}}$-III ${ }^{\mathrm{e}}$ siècle av. au $\mathrm{III}^{\mathrm{e}}-\mathrm{IV}^{\mathrm{e}}$ siècle de n. è., on retrouve des tessons de céramique hellénistique à vernis noir (marqueurs de la première phase de développement de la ville, entre la fin du IV et le $\mathrm{II}^{\mathrm{e}}$ siècle av. n. è.) sur des tombes de toute taille, et à n'importe quelle distance de la ville. Il semble notamment que la nécropole ait atteint son extension maximale vers le nord-ouest et vers le sud dès cette phase précoce. Dans la zone nord-est, le fait que la sépulture du Tall al-Zayir, datée des $\mathrm{I}^{\mathrm{er}}$-II ${ }^{\mathrm{e}}$ siècles de n. è., soit installée sur les vestiges d'une ancienne zone artisanale du $\mathrm{IV}^{\mathrm{e}}$ ou $\mathrm{III}^{\mathrm{e}}$ siècle av. n. è. peut accréditer l'idée d'une expansion tardive de la nécropole dans ce secteur.

Des facteurs sociaux ont pu jouer, au moins à certaines époques. Ceux-ci sont difficiles à identifier en l'état actuel des connaissances, faute d'un nombre suffisant de sépultures fouillées. Cependant, dans la zone située immédiatement au nord-est de la ville, la mise au jour d'une sépulture exceptionnellement riche au sein du Tall al-Zayir et la présence exclusive de tumulus de très grande taille pourraient indiquer qu'il y avait là, aux $\mathrm{I}^{\mathrm{er}}-\mathrm{II}^{\mathrm{e}}$ siècles de $\mathrm{n}$. è., une concentration de tombes élitaires. À cette époque, on peut supposer que les élites de Thaj aient favorisé cette zone proche de la ville, d'autant plus exclusive qu'elle était dépourvue de tombeaux plus anciens.

Bien qu'elle ne semble pas s'organiser en fonction d'axes de circulation, la nécropole était traversée par les voies d'accès à la ville, comme le montre la présence, dans 


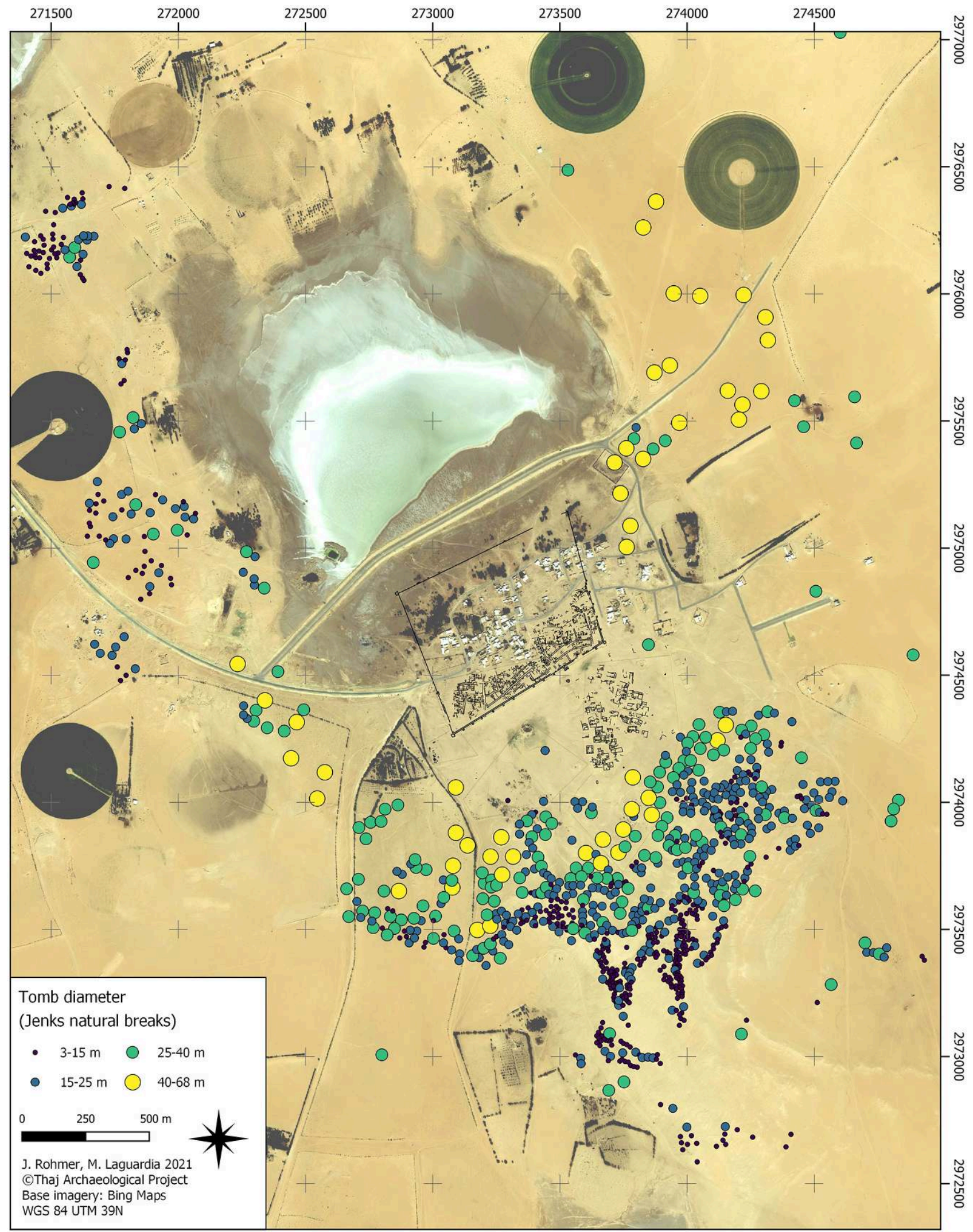

Figure 5. Distribution des structures tumulaires selon le diamètre (Thaj Archaeological Project. J. Rohmer, M. Laguardia 2021) / Distribution of tumulus structures according to their diameter (Thaj Archaeological Project. J. Rohmer, M. Laguardia 2021) 


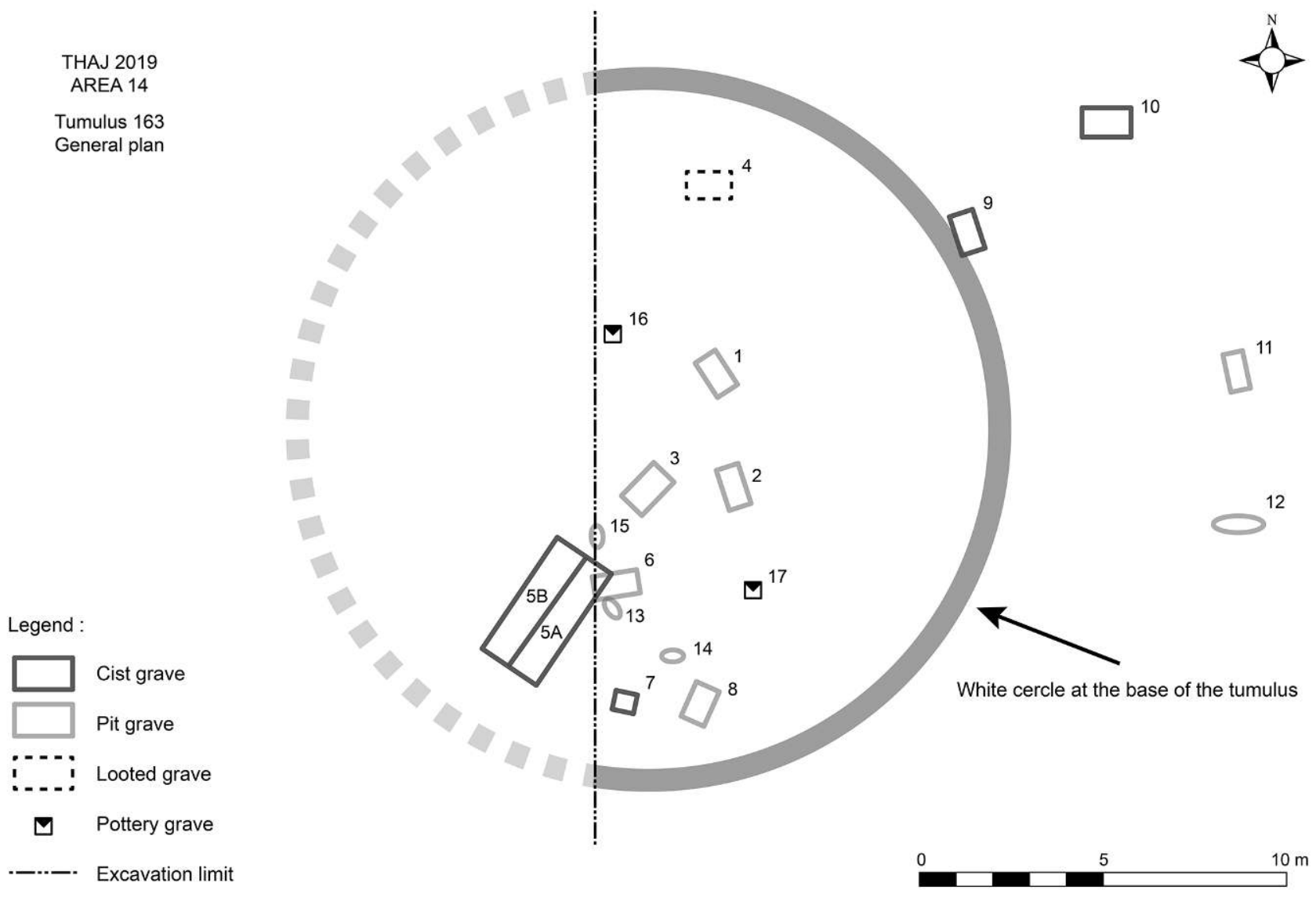

Figure 6. Plan du tumulus 163 avec la localisation des différents types de sépulture (Thaj Archaeological Project. M. Laguardia, P. Jaccard 2019) / Map of tumulus 163 showing the locations of the different types of graves (Thaj Archaeological Project. M. Laguardia, P. Jaccard 2019)

sa partie sud, d'un axe rectiligne nord-sud bordé de tombeaux et de bâtiments indéterminés (figure 2). Preuve de son importance symbolique, le paysage funéraire s'imposait donc aux voyageurs avant le paysage urbain. Outre cela, il est difficile de dire si la nécropole était fréquentée à des fins autres que funéraires. Dans la partie sud de la nécropole, la fouille du tumulus 71 a révélé que ce monument funéraire était construit sur une ancienne carrière, dont les déchets ont pu servir à habiller ses flancs. On ne peut cependant pas savoir si des carrières en activité coexistaient avec les tombes, ou si la nécropole a recouvert des carrières déjà désaffectées. Il est également possible que des carrières aient été créées pour la construction des tumulus. Quoi qu'il en soit, comme le montre l'exemple du Tall al-Zayir, installé sur un ancien atelier de potier, la frontière entre le monde des vivants et celui des morts a pu considérablement évoluer durant la longue histoire du site.

\section{Pratiques funéraires: une diversité de traitements}

Depuis 2017, le programme de recherche initié à Thaj a permis d'ouvrir huit secteurs de fouille dans la nécropole (figure 2), correspondant à deux cercles blancs, cinq tumulus, et une ciste isolée (annexe 1). Combinées aux résultats des travaux précédemment menés par la prospection danoise (1968) et le Département des antiquités saoudiens (1998-2001), ces fouilles permettent désormais de mieux appréhender la diversité des traitements funéraires au sein de la nécropole.

Les monuments qui ont été fouillés ont livré jusqu'à 19 sépultures individuelles (al-Hashash, 2006). Les premières analyses anthropologiques montrent la présence d'individus de tout âge et des deux sexes au sein d'un même monument funéraire. On observe cependant une certaine diversité des traitements funéraires (figure 6).

\section{Diversité des sépultures et des traitements funéraires}

Dans les monuments funéraires fouillés, les adultes et les immatures sont inhumés dans des cistes ou dans des fosses. Les cistes constituent le type de sépulture le plus fréquemment observé. Il s'agit de coffrages rectangulaires faits de blocs de calcaire taillés, liés par du mortier, et recouverts par des dalles de couverture (figure 7). Elles sont installées dans des fosses plus ou moins profondes, de sorte que la partie supérieure de la couverture peut soit être visible en surface, soit être enterrée. Certaines fosses peuvent être très profondes, révélant une volonté délibérée de dissimuler la ciste et son contenu. Parmi ce type de 

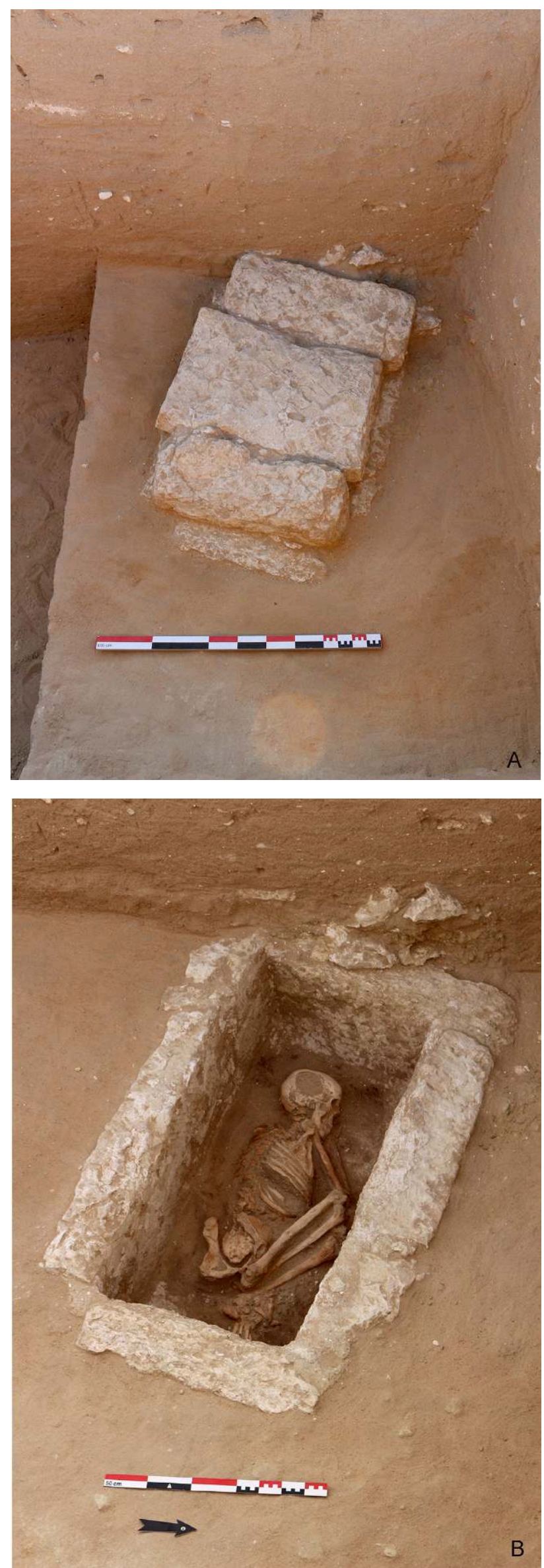

Figure 7. Ciste simple. A) trois dalles de pierre calcaire scellent la ciste. B) ciste rectangulaire de petites dimensions où un individu repose en décubitus latéral gauche avec les membres fléchis (Thaj Archaeological Project. M. Laguardia 2019) / Simple cist. A) three limestone slabs sealing the cist. $B$ ) small rectangular cist where an individual lies in the left lateral decubitus position with flexed limbs (Thaj Archaeological Project. M. Laguardia 2019) 
sépulture, il existe des cistes simples, de dimensions variées, qui comportent des dépôts individuels, tandis que d'autres possèdent une architecture plus complexe caractérisée par des partitions internes. Par exemple, la sépulture 5 du tumulus 163, situé dans la nécropole sud, est composée d'une double ciste rectangulaire dont chaque côté est compartimenté en deux ou trois parties par l'adjonction de blocs verticaux (figure 8). Cette configuration forme un ensemble de compartiments de petites dimensions qui servent à accueillir plusieurs morts de façon individuelle. La sépulture 5 présente également un "double fond", c'est-à-dire un niveau inférieur scellé par de gros blocs calcaires où des défunts ont été déposés.

Dans les cistes, les individus sont déposés en position latérale avec les membres fléchis, ou en position dorsale avec les membres en extension, en fonction de la dimension de la structure (annexe 1). La fouille de ces sépultures a révélé la présence d'un sédiment brun foncé au contact des ossements et, dans certain cas, les résidus d'un tissage ont été mis en évidence. Ces éléments suggèrent l'utilisation d'une enveloppe souple en matière périssable entourant le corps du défunt. De plus, des traces de coffrage, probablement du bois, ont été observées dans plusieurs cistes.
Des prélèvements ont été effectués et une analyse archéobotanique est en cours afin de déterminer la nature et la provenance du matériau.

Les défunts peuvent être accompagnés de mobilier funéraire, le plus souvent des parures composées de perles en pierres semi-précieuses. Deux sépultures en ciste fouillées par le Département des Antiquités saoudien ont livré un mobilier particulièrement riche : la sépulture du Tall al-Zayir, déjà évoquée plus haut, et la sépulture 1 du tumulus 193 (al-Hashash et al., 2001 ; al-Hashash, 2006). Dans la première, un enfant âgé de 5-6 ans reposait sur un lit funéraire à pieds anthropomorphiques plaqué en bronze et en plomb, orné de motifs méditerranéens ; le défunt était recouvert d'éléments ornementaux en or (masque, bandeaux, gant, et appliques circulaires), paré de nombreux bijoux en or, perles fines et pierres précieuses, et accompagné de vases en métal (Reeler et al-Shaikh, 2010). Dans certaines tombes fouillées plus récemment (sépultures 3 et 6 du cercle blanc 371), quelques perles (en améthyste, ambre et grenat) ayant échappé aux pilleurs ont été mises au jour. Elles trouvent des parallèles étroits dans la tombe d'Ayn Jawan, sur la côte du Golfe, datée entre le $\mathrm{I}^{\mathrm{er}}$ et le $\mathrm{III}^{\mathrm{e}}$ siècles de n. è. (al-Saud, 2010).

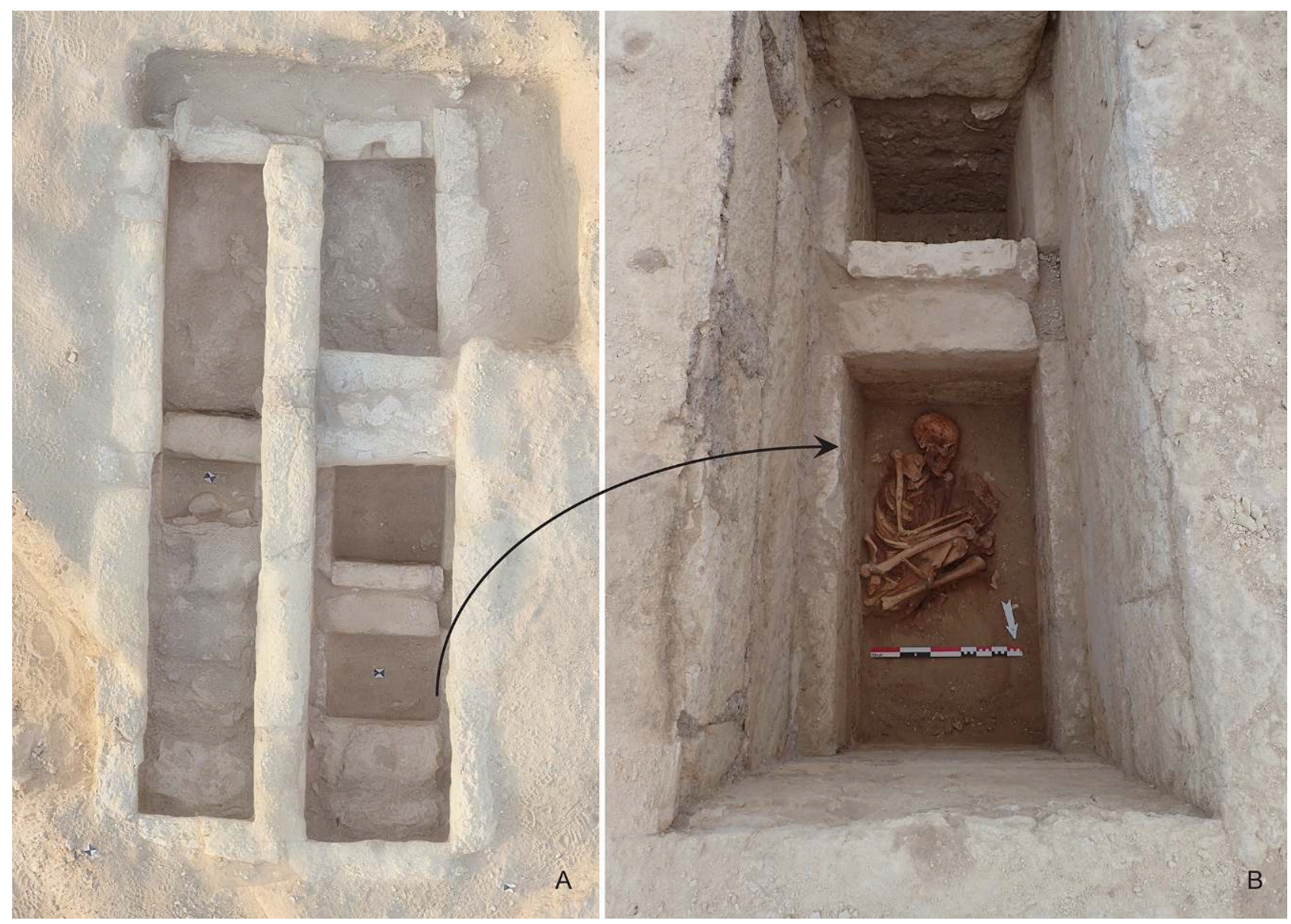

Figure 8. Ciste complexe. A) double ciste avec partition interne. B) individu découvert dans le compartiment inférieur de la ciste ou "double fond" (Thaj Archaeological Project. M. Laguardia, P. Courtaud 2021) / Complex cist. A) double cist with internal partition. B) individual discovered in the lower compartment of the cist (Thaj Archaeological Project. M. Laguardia, P. Courtaud 2021) 

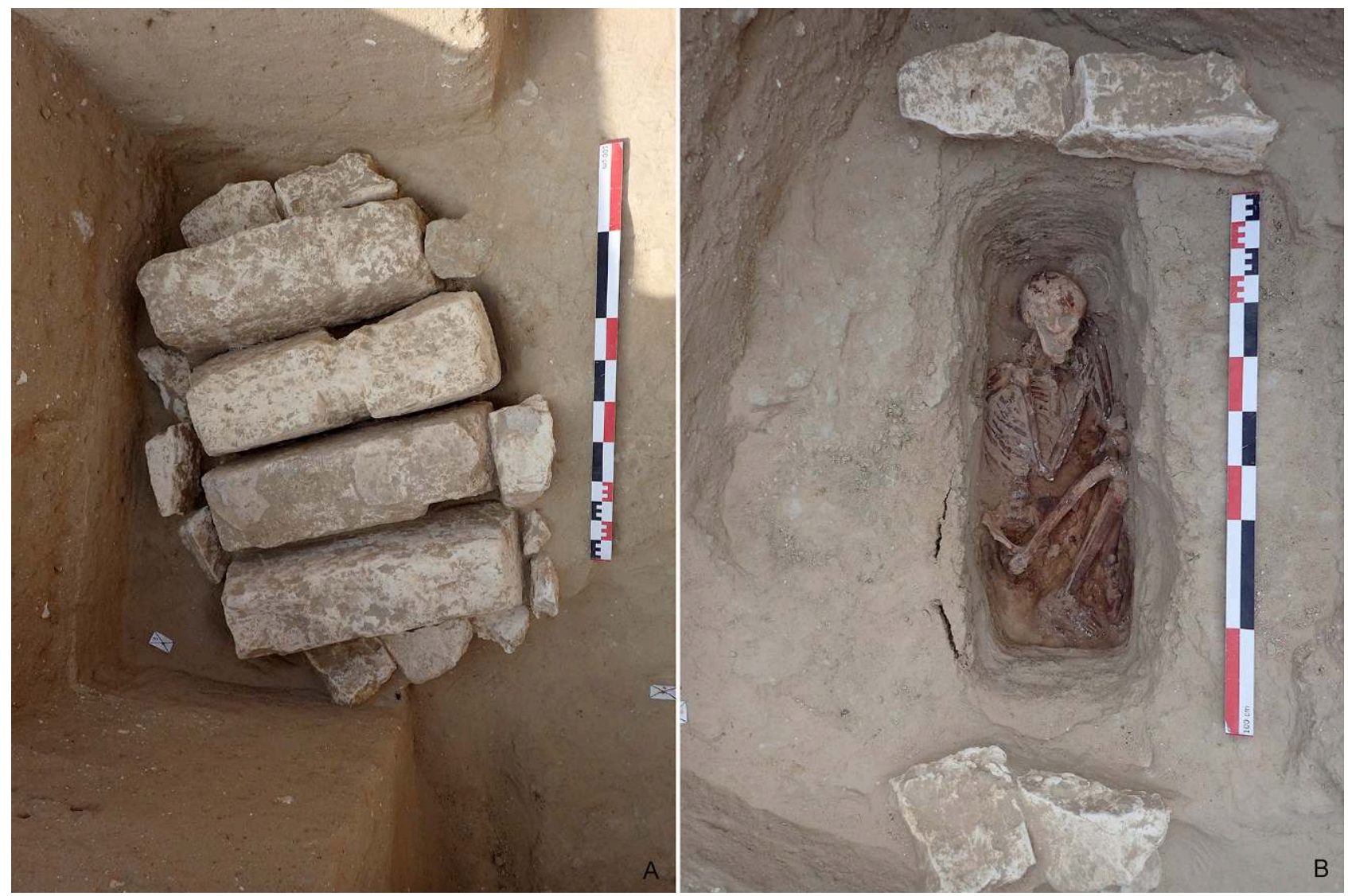

Figure 9. Tombe en fosse. A) dalles de pierre calcaire recouvrent la sépulture. B) fosse rectangulaire creusée dans le substrat naturel marneux où repose un individu en décubitus latéral gauche (Thaj Archaeological Project. M. Laguardia, 0. Munoz 2018) / Pit grave. A) limestone slabs covering the grave. B) rectangular pit dug into the natural marl substrate where an individual lies in the left lateral decubitus position (Thaj Archaeological Project. M. Laguardia, O. Munoz 2018)

Le deuxième type de sépulture observé est la tombe en fosse (figure 9). De petites dimensions, les fosses rectangulaires sont creusées dans le substrat naturel, rocheux ou marneux, et recouvertes par des dalles de calcaire taillé. Dans ce type de sépulture, les adultes sont déposés exclusivement en position latérale avec les membres fléchis (annexe 1). Dans certains cas, les individus sont inhumés avec du mobilier funéraire (collier de perles, bol en verre, brûle-encens cuboïde).

Ces différents types de sépulture et de traitement peuvent coexister au sein d'un même monument funéraire, comme le montrent les fouilles du cercle blanc 371 et du tumulus 163 . D'après les données disponibles, on n'observe pas de corrélation systématique entre le type de sépulture et de traitement d'une part, et le sexe ou l'âge des défunts d'autre part (à l'exception du cas particulier des individus périnatals). On n'observe pas non plus de corrélation chronologique, les différents types de sépulture et de traitement étant représentés dans les différents horizons chronologiques attestés. Dans le tumulus 193, fouillé par de Département des Antiquités saoudien, on constate une certaine corrélation entre le degré d'élaboration de la sépulture et la richesse du mobilier funéraire. Sur 19 tombes mises au jour, 18 étaient des sépultures en fosse et une en ciste, la seule à avoir livré un riche mobilier funéraire. Parmi les sépultures en fosse, seulement une a livré du mobilier, plus modeste. Malheureusement, cette corrélation ne peut pas être confirmée pour le moment par les nouvelles fouilles, car une grande partie des sépultures fouillées avaient été dépouillées de leur mobilier funéraire par des pillages. Il semble toutefois raisonnable de considérer, dans l'état actuel des connaissances, que le type de sépulture était avant tout lié à la richesse ou au statut social du défunt. De nouvelles données seront nécessaires pour confirmer ces observations.

\section{Le cas des décès en période périnatale}

D'après les fouilles réalisées, il apparait que des sujets périnatals étaient intégrés dans les enceintes funéraires, mais qu'ils bénéficiaient d'un traitement spécifique. Plusieurs sujets ont ainsi été mis au jour dans des fosses ovoïdes simples, creusées dans le sable (cercle blanc 371, tumulus 163). À deux reprises, des ossements d'individus périnatals ont été découverts dans des cistes pillées, mélangés à des ossements d'adultes. Les conditions ne permettent pas de déterminer si les défunts ont été déposés intentionnellement dans la même sépulture, mais cette éventualité ne peut être exclue. Au vu de la faible représentativité des ossements dans certains cas, un apport via le sédiment de remplissage est également envisageable. 


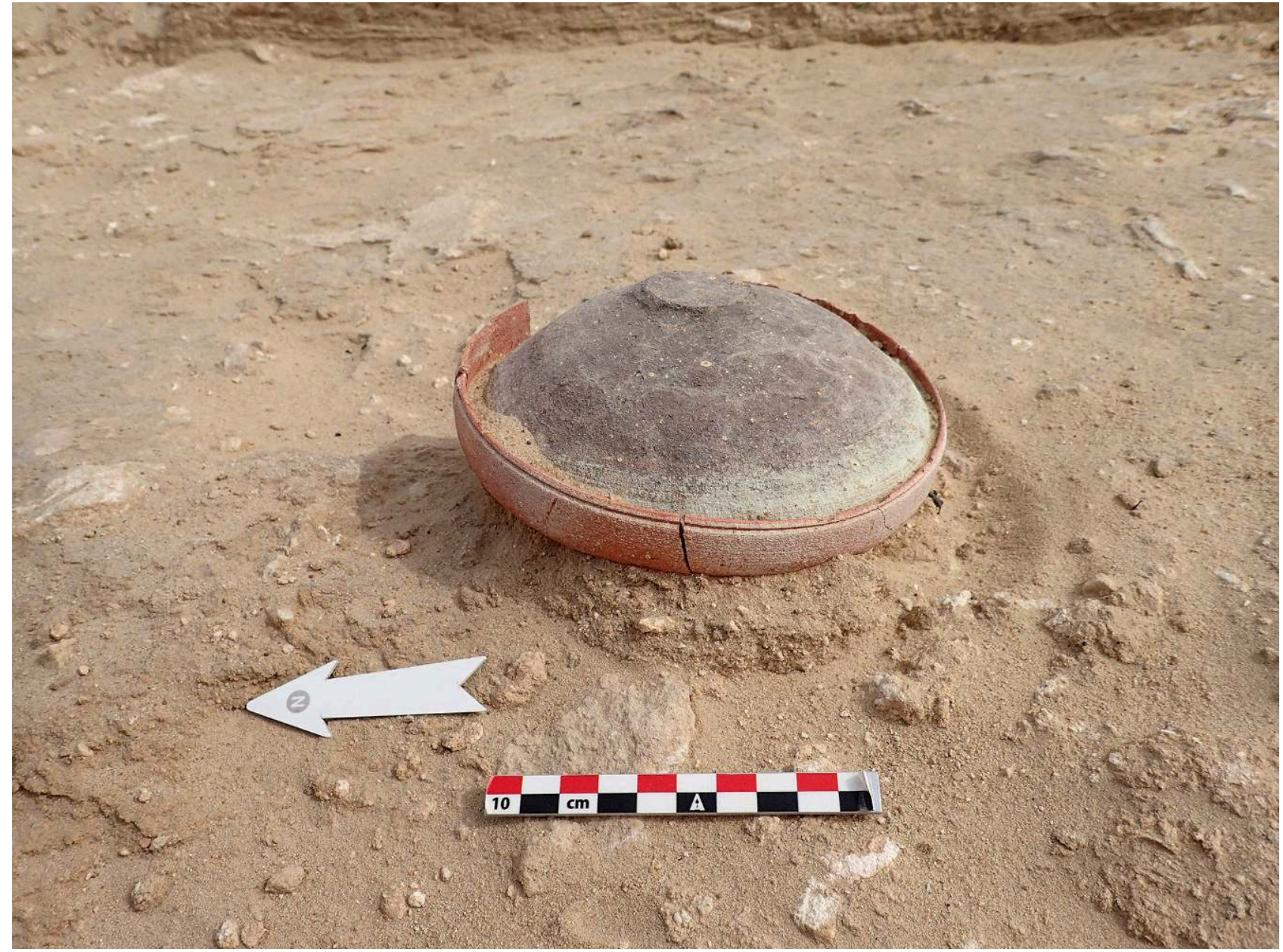

Figure 10. Réceptacle funéraire contenant les restes d'un fotus, retrouvé in situ (Thaj Archaeological Project. M. Laguardia 2019) / Pottery vessel found in situ, containing the remains of a foetus (Thaj Archaeological Project. M. Laguardia 2019)

Par ailleurs, la fouille du tumulus 163 a révélé deux exemples de fœtus déposés dans des vases en céramique. Ces fœtus, âgés respectivement de 4 et 5 mois lunaires ${ }^{4}$, avaient chacun été déposés dans un petit bol, recouvert d'un autre bol renversé (figure 10).

\section{La question des stèles funéraires}

Depuis les premières explorations du site, au début du $\mathrm{XX}^{\mathrm{e}}$ siècle, 27 inscriptions ont été retrouvées à Thaj (Rohmer et al., 2018 ; Sima, 2002). Ces inscriptions utilisent l'alphabet sud-arabique et transcrivent une langue sémitique locale encore mal caractérisée. On les qualifie de "hasaïtiques". Sur ces 27 inscriptions, 21 sont de nature funéraire. Il s'agit de stèles, soigneusement gravées et taillées, qui reprennent un formulaire standardisé indiquant le nom du défunt ou de la défunte, sa filiation et parfois son affiliation clanique ou tribale. Pour les défuntes, on observe l'usage récurrent de filiations matrilinéaires.

Malheureusement, aucune de ces inscriptions funéraires n'a encore été retrouvée in situ, de sorte que l'on ne peut savoir comment elles étaient disposées par rapport aux tombes ${ }^{5}$. Le fait qu'elles ne mentionnent qu'un seul défunt suggère qu'elles étaient associées à des sépultures individuelles. Comme l'avait déjà remarqué D. Potts (1990 : 7374), il est possible qu'elles n'aient pas eu vocation à être visibles, mais qu'elles aient été enterrées au sein des monuments funéraires. On peut cependant aussi imaginer qu'elles étaient associées à des tombes isolées, ou que, comme dans les dernières phases de la période Tylos à Bahreïn, elles aient été rassemblées par groupes en périphérie des monuments funéraires (Andersen et Salman, $2006: 118$ ). Seule la découverte de stèles in situ permettra de répondre à ces interrogations.
4 Détermination de l'âge au décès à partir de la mesure des os longs d'après Fazekas et Koza, 1978.
5 La seule inscription retrouvée en fouille était remployée dans le mur d'une ciste plus tardive (Bibby, 1973). 


\section{Un lien maintenu au cours des siècles}

Si les tombes étaient destinées à accueillir des défunts de tout âge, l'organisation et le fonctionnement de ces structures complexes restent toutefois à préciser sur la longue période d'utilisation de la nécropole.

\section{Des monuments utilisés sur de longues périodes}

Les datations par le radiocarbone effectuées sur des vestiges osseux (collagène) ou sur des charbons provenant du mortier des cistes ont révélé que certains monuments funéraires pouvaient être utilisés pendant plusieurs siècles (tableau 2). Par exemple, le cercle blanc 371, localisé dans la nécropole sud, a livré des cistes et des fosses datées entre les $\mathrm{II}^{\mathrm{e}}-\mathrm{I}^{\mathrm{er}}$ siècles av. et les $\mathrm{II}^{\mathrm{e}}-\mathrm{III}^{\mathrm{e}}$ siècles de n. è. On observe également l'implantation de nouvelles sépultures recoupant des tombes plus anciennes (tumulus 163). L'ajout de nouvelles sépultures a pu impliquer des remaniements au sein du monument funéraire et, parfois, des restaurations de la structure. En effet, trois niveaux de fragments calcaires blancs ont été mis en évidence dans la stratigraphie du cercle blanc 371, indiquant la volonté de préserver l'aspect et la cohérence de l'enceinte sur sa très longue période d'utilisation. Ces remaniements successifs témoignent d'un investissement collectif particulièrement fort et durable dans l'entretien des différents monuments de la nécropole.

\section{Les dépôts associés aux cérémonies funéraires}

Les prospections ont révélé la présence d'une grande quantité de céramique de surface sur toute l'étendue de la nécropole de Thaj. L'étude de cette céramique est encore en cours, mais des résultats préliminaires indiquent la présence de divers types de vases (bols de différentes tailles, jarres, marmites), majoritairement produits localement mais parfois importés de Mésopotamie et de Méditerranée orientale. Parmi ces importations, la présence récurrente de bols et de coupes en céramique à vernis noir (importée de Grèce ou de Méditerranée orientale) ou glaçurée (importée de l'aire mésopotamienne) suggère la tenue de banquets. En proportion, cette céramique de prestige semble nettement surreprésentée dans la nécropole, ce qui souligne l'importance symbolique de ces cérémonies et de ces dépôts.

En fouille, les travaux effectués sur le tumulus 163 ont permis la découverte de 8 dépôts de vases in situ, de formes et de tailles variées, disposés à l'endroit ou à l'envers et parfois empilés. Ce matériel, localisé à l'extérieur des sépultures, dans l'enceinte circulaire du tumulus, évoque de la vaisselle laissée sur place après un repas funéraire, mais pourrait aussi correspondre à des dépôts alimentaires en hommage au(x) défunt(s). Ces dépôts sont associés à des restes botaniques (noyaux de dattes carbonisés, charbon de bois indéterminé) et fauniques (os de dromadaire, poissons) ainsi qu'à des brûle-encens, témoignant de la consommation ou de dépôts de nourriture et de l'éventuelle utilisation d'aromates lors des cérémonies funéraires. L'étude de ces restes est en cours et permettra de mieux caractériser la nature des produits utilisés. Des vases ont également été retrouvés dans les tranchées de fondation des cistes ou déposés à l'envers sur les dalles de couverture (Laguardia, 2021).

Toutes ces informations suggèrent que, au moins dans certains cas, le rituel funéraire comportait un repas et que des dépôts alimentaires pouvaient se dérouler lors de cérémonies secondaires.

\begin{tabular}{|c|c|c|c|c|c|c|}
\hline Tombe no & Sép. no & Type de sépulture & Lab \# & Nature & $\hat{A} g e^{14} C$ & Date Cal, 2 $\sigma$ IntCal 13 \\
\hline \multirow{8}{*}{ CB 371} & 1 & \multirow{3}{*}{ Ciste } & UBA-36874 & \multirow{9}{*}{ Charbon } & $2135 \pm 26$ & 209-88 av. n. è. \\
\hline & 2 & & UBA-36875 & & $2096 \pm 30$ & 194-46 av. n. è. \\
\hline & 3 & & UBA-36877 & & $1872 \pm 34$ & $69-230$ de n. è. \\
\hline & 4 & Ciste à deux compartiments & UBA-40163 & & $2058 \pm 32$ & 170 av. n. è.- 16 de n. è. \\
\hline & 6 & \multirow{2}{*}{ Fosse rectangulaire } & UBA-40165 & & $1844 \pm 41$ & $71-254$ de n. è. \\
\hline & 7 & & UBA-40166 & & $2046 \pm 24$ & 159 av. n. è.- 18 de n. è. \\
\hline & 8 & Ciste détruite & UBA-40161 & & $1915 \pm 26$ & 24-133 de n. è. \\
\hline & 9 & Ciste & UBA-42796 & & $2062 \pm 26$ & 166-19 av. n. è. \\
\hline Tum. 340 & 1 & $\begin{array}{l}\text { Structure rectangulaire } \\
\text { (indéterminée) }\end{array}$ & UBA-36878 & & $2339 \pm 26$ & 433-369 av. n. è. \\
\hline Tum. 163 & 5 & $\begin{array}{c}\text { Double ciste avec } \\
\text { compartiments internes }\end{array}$ & UBA-42804 & $\begin{array}{c}\text { Os } \\
\text { (collagène) }\end{array}$ & $2077 \pm 29$ & 181-37 av. n. è. \\
\hline Tum. 190 & 5 & Ciste & UBA-42807 & Charbon & $1935 \pm 29$ & 4-128 de n. è. \\
\hline
\end{tabular}

Tableau 2. Datations par le radiocarbone (AMS) provenant des sépultures de Thaj / Radiocarbon dates (AMS) obtained for the Thaj burials 


\section{Pillages fréquents}

Depuis ou même durant l'Antiquité, les tombes de Thaj ont fait l'objet de nombreuses perturbations. Des indices ont montré que les pillages sont ciblés et qu'ils surviennent, dans certains cas documentés, peu de temps après les dépôts des défunts. En effet, plusieurs sépultures pillées ont livré des segments anatomiques encore en connexion mais éloignés de leur position d'origine. Les pillards se seraient introduits dans la tombe alors que le processus de décomposition n'était pas encore achevé. De plus, certaines perturbations observées exclusivement au niveau du thorax et du bassin permettent de reconstituer les gestes des pilleurs, qui ciblent le mobilier funéraire (bijoux, ceinture, etc.). Ces données permettent d'affirmer que les pilleurs connaissaient l'emplacement des nouvelles sépultures et la richesse du mobilier qui pouvait accompagner les défunts.

Des méthodes efficaces ont probablement été mises au point pour s'introduire dans les sépultures. À plusieurs reprises, le seul élément permettant de distinguer une sépulture pillée est une petite cavité au niveau des blocs de couverture. Un seul bloc est en partie détruit, ce qui forme une brèche dans la sépulture pour atteindre l'intérieur, tout en conservant l'architecture funéraire. L'étroitesse de certaines ouvertures suggère l'utilisation d'outils spécifiques ou même l'intervention d'enfants (figure 11). La population semble avoir eu conscience de ces pillages puisqu'on retrouve des aménagements manifestement destinés à lutter contre la violation des tombes : cistes à "double fond"; couverture comportant deux niveaux de blocs ; implantation d'une fosse sépulcrale dans des sondages très profonds. Ces caractéristiques révèlent une volonté de protection des morts de la part des inhumants.

\section{Conclusion}

En conclusion, les travaux archéologiques menés jusqu'ici dans la nécropole de Thaj permettent de mieux appréhender trois modalités fondamentales du rapport entre les vivants et les morts dans ce qui fut sans doute la principale ville antique d'Arabie orientale, entre les $\mathrm{IV}^{\mathrm{e}}$-III' ${ }^{\mathrm{e}}$ av. et le IV siècle de n. è. En premier lieu, on observe la constitution d'un imposant paysage funéraire autour de la ville, à travers la construction de plus d'un millier de monuments funéraires. Répartis en deux grands types (tumulus et cercles blancs), ces monuments présentent en réalité des principes de construction communs et un même habillage de fragments de calcaire blancs qui accentue leur marque sur le paysage. Leur répartition privilégie des impératifs de visibilité, mais s'explique également par des facteurs chronologiques et sociaux.

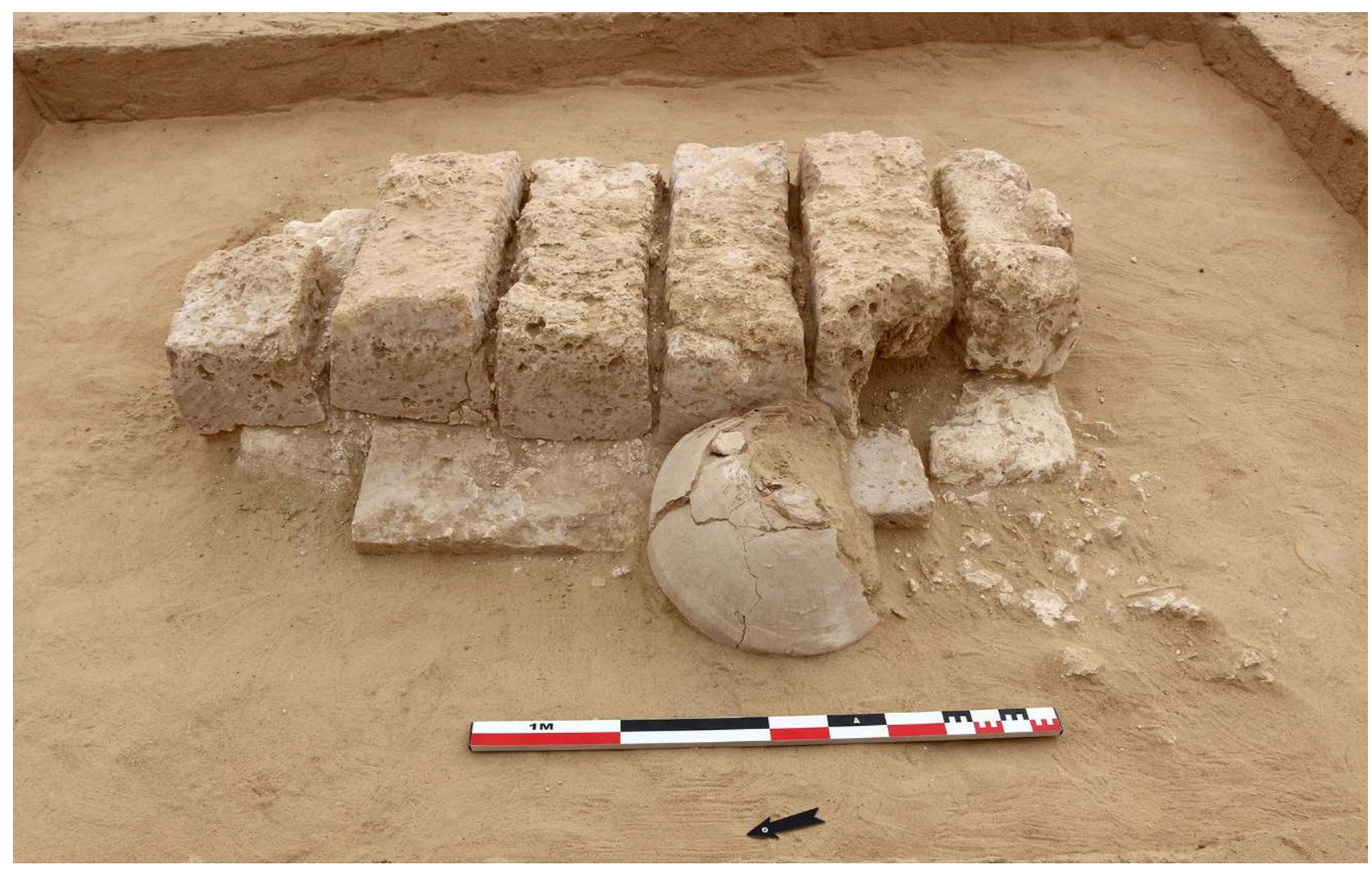

Figure 11. Couverture de dalles conservée à l'exception d'une brèche au sud-ouest qui a permis d'atteindre l'intérieur de la ciste (Thaj Archaeological Project. M. Laguardia, P. Jaccard, P Dumas-Lattaque 2019) / Covering slabs preserved except for a hole in the south-west corner, which gave access to the interior of the cist (Thaj Archaeological Project. M. Laguardia, P. Jaccard, P. Dumas-Lattaque 2019) 
En deuxième lieu, il est désormais possible de mieux appréhender la diversité des traitements funéraires. L'ensemble des classes d'âge semble avoir eu accès aux espaces sépulcraux, y compris les individus décédés en période périnatale. Ceux-ci faisaient cependant l'objet d'un traitement spécifique (inhumation en pleine terre sans dalles de couverture, ou dans des vases en céramique). Quant aux adultes, ils faisaient l'objet de traitements différenciés (inhumations en position fléchie dans des fosses couvertes, ou en décubitus dans des cistes plus ou moins complexes, présence d'un matériel funéraire plus ou moins riche et abondant) qui tiennent probablement davantage à des écarts de richesse et de statut social qu'à une évolution chronologique. Quant à la question du marquage des sépultures, elle reste problématique : bien que l'on connaisse une série de stèles funéraires inscrites dans la graphie et la langue locale, la question de leur emplacement et de leur visibilité reste en suspens.

Enfin, les prospections et les fouilles révèlent la persistance à travers le temps du lien entre les vivants et les morts, que ce soit à travers l'utilisation et l'entretien des monuments funéraires sur plusieurs siècles, ou à travers la tenue de cérémonies funéraires. Tenues au moment de l'inhumation ou plus tard, à titre commémoratif, ces cérémonies pouvaient impliquer des banquets, dont la valeur symbolique est soulignée par l'usage d'une vaisselle de prestige. Cependant, l'importance accordée au soin des morts ne semble pas avoir empêché les pillages, dont les plus précoces semblent avoir eu lieu dès l'Antiquité, très peu de temps après les inhumations.

Les résultats préliminaires exposés ci-dessus permettent donc déjà de mieux comprendre l'importance et les modalités des pratiques funéraires dans la ville de Thaj. Ils doivent toutefois encore être complétés et enrichis par une série d'études en cours. L'étude biologique permettra notamment de préciser les modalités de recrutement des monuments funéraires, y compris à l'aide d'analyses paléogénomiques visant à mettre en évidence d'éventuels liens de parenté. Les études archéobotanique et archéozoologique permettront quant à elles de préciser la nature des aliments consommés ou offerts lors des cérémonies funéraires, tandis que l'étude céramologique permettra d'affiner la chronologie de la nécropole et de mieux comprendre la nature des activités humaines qui s'y tenaient. Enfin, de nouvelles fouilles sont prévues afin d'étendre le corpus d'étude et augmenter sa représentativité, tant spatiale que sociale ou chronologique.

\section{Références}

Andersen SF, Salman MI (2006) The Tylos burials in Bahrain. Proceedings of the Seminar for Arabian Studies 36:111-124

Bibby G (1973) Preliminary Survey in East Arabia. Reports of the Danish Archaeological Expedition to the Arabian Gulf, Vol. 2. Jutland Archaeological Society Publications 12, Gyldendal, Copenhagen

Bowen LBR, Albright WF, Matson FR et al (1950) The Early Arabian necropolis of Ayn Jawan: A Pre-Islamic and Early
Islamic site on the Persian Gulf. Bulletin of the American Schools of Oriental Research, Supplementary Studies 7/9:1-70

Bruzek J (2002) A method for visual determination of sex, using the human hip bone. American Journal of Physical Anthropology 117:157-68 [https://doi.org/10.1002/ajpa.10012]

Fazekas IG, Koza F (1978) Forensic Foetal Osteology. Akadémiai Kiadó, Budapest

al-Hashash A (2005) Report on the excavation of Thaj. Atlal 18:19-21

al-Hashash A (2006) Brief report on the excavations at Thaj. Atlal 19:15-19

al-Hashash A, al-Saif ZA, al-Sanna SH et al (2002) Report on the excavations of Thaj. Atlal 17:17-21

al-Hashash A, al-Zayer WA, al-Saif ZA, et al (2001) Report on the archaeological excavations at Thaj. Atlal 16:23-26

Højlund F (2007) The Burial Mounds of Bahrain. Social complexity in Early Dilmun. Jutland Archaeological Society Publications 58, Aarhus

Laguardia M (2021) Repas des morts, repas des vivants : analyse des dépôts funéraires de la nécropole de Thaj (Arabie Saoudite). In: Bacoup P, Caurette N, Laurent A-S et al (dir) À table ! De l'approvisionnement au dernier repas. Regards croisés sur l'archéologie de l'alimentation, Archéodoct 15, Éditions de la Sorbonne, Paris [https://doi.org/10.4000/books. psorbonne.107065]

Laguardia M, Munoz O, Rohmer J (2019) The necropolis of Thaj (Eastern Province of Saudi Arabia): An archaeological and anthropological approach. Proceedings of the Seminar for Arabian Studies 49:199-206

Mandaville JP (1963) Thaj: A Pre-Islamic site in Northeastern Arabia. Bulletin of the American Schools of Oriental Research 172:9-20

Moorrees CFA, Fanning EA, Hunt EE (1963a) Age variation of formation stages for ten permanent teeth. Journal of dental Research 42(6):1490-1502 [https://doi.org/10.1177/0022034 5630420062701]

Moorrees CFA, Fanning EA, Hunt EE (1963b) Formation and resorption of three deciduous teeth in children. American Journal of Physical Anthropology 21(2):205-213 [https://doi. org/10.1002/ajpa.1330210212]

Murail P, Bruzek J, Houët F et al (2005) DSP: a probabilistic sex diagnosis tool using worldwide variability in hip bone measurements. Bulletins et Mémoires de la Société d'Anthropologie de Paris 17:167-76 [https://doi.org/10.4000/bmsap.1157]

Potts DT (1990) The Arabian Gulf in Antiquity, vol 2. From Alexander the Great to the Coming of Islam. Clarendon Press, Oxford

Potts DT (1993) The sequence and chronology of Ayn Jawan. In Finkbeiner U (ed) Materialien zur Archäologie der Seleukidenund Partherzeit im südlichen Babylonien und im Golfgebiet. Wasmuth, Tübingen, pp 111-126

Reeler C, al-Shaikh N (2010) The tomb of Thaj. In: al-Ghabban AI, André-Salvini B, Demange F et al (eds) Roads of Arabia: Archaeology and History of the Kingdom of Saudi Arabia. Musée du Louvre/Somogy Art Publishers, Paris, pp 392-397

Robin CJ (2016) Gerrha d'Arabie, cité séleucide. Syria III:223250 [https://doi.org/10.4000/syria.5319] 
Robin CJ, Prioletta A (2013) Nouveaux arguments en faveur d'une identification de la cité de Gerrha avec le royaume de Hagar (Arabie orientale). Semitica et Classica 6:131-185 [https://doi.org/10.1484/J.SEC.1.103730]

Rohmer J (2019) Aux marges des mondes hellénistique, parthe et sassanide : la ville caravanière de Thaj (Arabie orientale). Revue Archéologique 67 (Bulletin de la Société française d'archéologie classique LXIX):167-176

Rohmer J, al-Jallad A, al-Hajiri M et al (2018) The Thaj archaeological project: results of the first field season. Proceedings of the Seminar for Arabian Studies 48:287-302

Salman MI, Andersen SF (2009) The Tylos burials in Bahrain. Vol 2. The Hamad Town DS3 and Shakhoura Cemeteries. Culture and National Heritage, Kingdom of Bahrain

al-Saud AS (2010) Ayn Jawan. In: al-Ghabban AI, André-Salvini B, Demange F et al (eds) Roads of Arabia: Archaeology and History of the Kingdom of Saudi Arabia. Musée du Louvre/ Somogy Art Publishers, Paris, pp 398-403

Scheuer L, Black S (2000) Developmental Juvenile Osteology. Elsevier Academic Press, San Diego

Schmitt A (2005) Une nouvelle méthode pour estimer l'âge au décès des adultes à partir de la surface sacro-pelvienne iliaque. Bulletins et Mémoires de la Société d'Anthropologie de Paris 17:89-101 [https://doi.org/10.4000/bmsap.943]

Sima A (2002) Die hasaitischen Inschriften. In: Nebes N (ed) Neue Beiträge zur Semitistik: erstes Arbeitstreffen der Arbeitsgemeinschaft Semitistik in der Deutschen Morgenländischen Gesellschaft vom 11. bis 13. September 2000 an der FriedrichSchiller-Universität Jena (Jenaer Beiträge zum Vorderen Orient, 5). Harrassowitz, Wiesbaden, pp 167-200

Zarins J, Mughannum AS, Kamal M (1984) Excavations at Dhahran South. The tumuli field. A preliminary report. Atlal 8:25-54 
Annexe 1. Résumé des données sur le traitement funéraire dans les tombes fouillées à Thaj

\begin{tabular}{|c|c|c|c|c|c|c|c|c|c|c|c|c|}
\hline Tombe & Sep. & Type de sépulture & $\begin{array}{c}\text { Dalles de } \\
\text { couverture }\end{array}$ & Orientation & Restes squelettiques & Âge (1) & Sexe (2) & Position & Contenant & Mobilier & Pillage & Référence \\
\hline \multirow{11}{*}{ СВ 371} & 1 & \multirow{2}{*}{ Ciste } & \multirow{6}{*}{ non } & \multirow{3}{*}{$\mathrm{N} / \mathrm{S}$} & Fragments & \multicolumn{4}{|c|}{ Non observable } & & \multirow{3}{*}{ Oui } & \multirow{3}{*}{$\begin{array}{l}\text { Laguardia M, Munoz O (2018) Zone 3: Excavation of a "White Circle" grave. } \\
\text { In: Rohmer } \mathrm{J} \text { (ed) Mission archéologique de Thaj. Rapport 2017/2018. Non } \\
\text { publié, pp 88-112. }\end{array}$} \\
\hline & 2 & & & & & Adulte & Masculin & Décubitus & Non observable & Aucun & & \\
\hline & 3 & Ciste sans dalles de couverture & & & Individu perturbé & $20-29$ ans & Féminin & Non observable & $\begin{array}{l}\text { Coffrage en bois? } \\
\text { Lit funéraire? }\end{array}$ & $\begin{array}{l}\text { Perles semi-précieuses, } \\
\text { objet en bronze }\end{array}$ & & \\
\hline & \multirow{2}{*}{4} & \multirow{2}{*}{ Ciste à deux compartiments } & & \multirow{3}{*}{$\mathrm{NO} / \mathrm{SE}$} & & Adulte & \multirow{2}{*}{\multicolumn{3}{|c|}{ Non observable }} & Aucun & \multirow{3}{*}{ Oui } & \multirow{7}{*}{$\begin{array}{l}\text { Laguardia M, Munoz O, Courtaud P (2019) Area 3: Excavation of a "White } \\
\text { Cirle" funerary structure. In: Rohmer J. (ed) Mission archéologique de Thaj } \\
\text { (Arabie Saoudite). Rapport des campagnes 2018-2019. Non publié, pp 121-170. }\end{array}$} \\
\hline & & & & & Fragments & Immature & & & & Aucun & & \\
\hline & 5 & Ciste & & & & & & Non observable & & & & \\
\hline & 6 & \multirow{2}{*}{ Fosse rectangulaire } & \multirow{2}{*}{ oui } & \multirow{2}{*}{$\mathrm{O} / \mathrm{E}$} & \multirow{2}{*}{ Individu complet } & Adulte & Féminin & \multirow{2}{*}{$\begin{array}{l}\text { Décubitus latéral gauche } \\
\text { avec les membres fléchis }\end{array}$} & Enveloppe souple & $\begin{array}{l}\text { Perles semi-précieuses, pâte de } \\
\text { verre et pierre, bol en verre }\end{array}$ & \multirow{2}{*}{ Non } & \\
\hline & 7 & & & & & $4-8$ ans & Indet. & & Aucun & Aucun & & \\
\hline & 8 & Ciste détruite & non & $\begin{array}{c}\text { Non } \\
\text { observable }\end{array}$ & Fragments & Adulte & \multicolumn{3}{|c|}{ Non observable } & $\begin{array}{l}\text { Fragments de bois et } \\
\text { éléments en fer }\end{array}$ & Oui & \\
\hline & 9 & Ciste & oui & \multirow[b]{2}{*}{$\mathrm{NO} / \mathrm{SE}$} & \multirow[b]{2}{*}{ Individu complet } & $30-50$ ans & Féminin & $\begin{array}{l}\text { Décubitus latéral gauche } \\
\text { avec les membres flechis }\end{array}$ & Enveloppe souple & \multirow[b]{2}{*}{ Aucun } & \multirow[b]{2}{*}{ Non } & \\
\hline & 10 & Fosse ovoide en pleine terre & non & & & $0-1$ an & Indet. & $\begin{array}{l}\text { Décubitus latéral droit } \\
\text { avec les membres fléchis }\end{array}$ & Aucun & & & $\begin{array}{l}\text { Laguardia M, Munoz O (2018) Zone 3: Excavation of a "White Circle" grave. } \\
\text { In: Rohmer I (ed) Mission archéologique de Thaj. Rapport 2017/2018. Non } \\
\text { publié, pp } 88 \text {-112. }\end{array}$ \\
\hline Tum 340 & 1 & $\begin{array}{c}\begin{array}{c}\text { Structure rectangulaire } \\
\text { (indéterminé) }\end{array} \\
\end{array}$ & non & \multicolumn{7}{|c|}{ Non observable } & Oui & $\begin{array}{l}\text { al-Hajiri M (2018) Report on Area 5. In: Rohmer J (ed) Mission archéologique } \\
\text { de Thaj. Rapport 2017/2018. Non publié, pp 133-136. }\end{array}$ \\
\hline Tum 607 & - & Sépultures islamiques & - & - & - & - & - & - & - & - & - & $\begin{array}{l}\text { Coudrin A (2018) Thaj 2017, Zone 4. In: Rohmer J (ed) Mission archéologique } \\
\text { de Thaj. Rapport 2017/2018. Non publié, pp 113-132. }\end{array}$ \\
\hline Tum 71 & 1 & Ciste & $\begin{array}{c}\text { oui, } \\
\text { en partie }\end{array}$ & & & & & Non fouillée & & & Oui & $\begin{array}{l}\text { Courtaud P, Creissen T (2019) Fouille de la zone 7. In: Rohmer J (ed) Mission } \\
\text { archéologique de Thaj (Arabie Saoudite). Rapport des campagnes 2018-2019. } \\
\text { Non publié, pp 187-222. }\end{array}$ \\
\hline \multirow{13}{*}{ Tum 163} & 1 & \multirow{4}{*}{ Fosse rectangulaire } & \multirow{3}{*}{ non } & $\mathrm{NO} / \mathrm{SE}$ & Individu perturbé & Adulte & - & Non observ & & Aucun & & \\
\hline & 2 & & & $\mathrm{~N} / \mathrm{S}$ & Fragments & Jeune adulte & & Non observable & & Bague en métal & & \\
\hline & 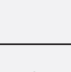 & & & & & Immature & & & & & & \\
\hline & 3 & & $\begin{array}{l}\text { oui, } \\
\text { en partie }\end{array}$ & $\mathrm{NE} / \mathrm{SO}$ & Individu perturbé & Adulte & - & $\begin{array}{l}\text { Décubitus latéral gauche } \\
\text { avec les membres fléchis }\end{array}$ & Aucun & Aucun & & \\
\hline & 4 & Ciste détruite & & $\begin{array}{c}\text { Non } \\
\text { observable }\end{array}$ & Fragments & - & & & bsservable & & Oui & \\
\hline & & & & & (5A) Individu perturbé & $20-29$ ans & Masculin & \begin{tabular}{|c|}
$\begin{array}{c}\text { Décubitus, membres sup. et inf. } \\
\text { en extension }\end{array}$ \\
\end{tabular} & Aucun & & & Laguardia M, Jaccard P, Courtaud P (2020) Area 14: excavation of tumulus \\
\hline & & & & & (5A) Fragments & Immature & & Non observable & & & & $\begin{array}{l}\text { no. 163. In Rohmer J (ed) Mission archéologique de Thaj. Rapport des campagnes } \\
\text { 2019/2020. Non publié }\end{array}$ \\
\hline & 5 & $\begin{array}{l}\text { Double ciste } \\
\text { avec compartiments internes }\end{array}$ & non & $\mathrm{NE} / \mathrm{SO}$ & $\begin{array}{l}\text { (5Bsup) } \\
2 \text { individus nerturbés }\end{array}$ & $30-40$ ans & Masculin & Non observ & & Aucun & & \\
\hline & & & & & (5Binf) Individu complet & $\begin{array}{c}\text { Adulte } \\
20-49 \text { ans }\end{array}$ & Féminin & $\begin{array}{l}\text { Décubitus latéral gauche } \\
\text { avec les membres fléchis }\end{array}$ & Enveloppe souple & & Non & \\
\hline & & & & & (5Binf) Fragments & 1-6 mois & - & & Non observable & & Oui & \\
\hline & 6 & Fosse rectangulaire & oui & $\mathrm{E} / \mathrm{O}$ & Individu complet & $20-29$ ans & Féminin & $\begin{array}{l}\text { Décubitus latéral gauche } \\
\text { avec les membres fléchis }\end{array}$ & Aucun & Aucun & Non & \\
\hline & 7 & Ciste & non & $\mathrm{NE} / \mathrm{SO}$ & Fragment & Immature & - & Non observ & & & Oui & \\
\hline & 8 & Fosse rectangulaire & non & $\mathrm{NE} / \mathrm{SO}$ & & & & Non fouillée & & & & \\
\hline
\end{tabular}

(1) Pour les individus immatures, détermination à partir de la minéralisation des dents (Moorrees et al., 1963a ; 1963b), la fusion des épiphyses et la longueur des os longs (Scheuer \& Black, 2000a). Pour les adultes, détermination à partir de la surface sacro-pelvienne iliaque (Schmitt, 2005).

(2) Détermination à partir de l'os coxal grâce aux méthodes morphologique (Bruzek, 2002) et métrique (Murail et al., 2005). 


\begin{tabular}{|c|c|c|c|c|c|c|c|c|c|c|c|c|}
\hline Tombe & Sep. & Type de sépulture & $\begin{array}{c}\begin{array}{c}\text { Dalles de } \\
\text { couverture }\end{array} \\
\end{array}$ & Orientation & Restes squelettiques & Âge (1) & Sexe (2) & Position & Contenant & Mobilier & Pillage & Référence \\
\hline \multirow{9}{*}{ Tum 163} & 9 & \multirow{2}{*}{ Ciste } & \multirow{6}{*}{ non } & $\mathrm{N} / \mathrm{s}$ & Fragments & \multirow{3}{*}{\multicolumn{4}{|c|}{ Non observable }} & \multirow{9}{*}{ Aucun } & \multirow{5}{*}{ Oui } & \multirow{9}{*}{$\begin{array}{l}\text { Laguardia M, Jaccard P, Courtaud P (2020) Area 14: excavation of tumulus } \\
\text { no. 163. In Rohmer J Jed) Mission archéologique de Thaj. Rapport des campagnes } \\
\text { 2019/2020. Non publie. }\end{array}$} \\
\hline & 10 & & & $\mathrm{E} / \mathrm{O}$ & Aucun & & & & & & & \\
\hline & 11 & Fosse rectangulaire & & $\mathrm{N} / \mathrm{S}$ & Aucun & & & & & & & \\
\hline & 12 & \multirow{4}{*}{ Fosse ovoide } & & $\mathrm{E} / \mathrm{O}$ & Fragments & Adulte & \multirow{2}{*}{\multicolumn{3}{|c|}{ Non observable }} & & & \\
\hline & 13 & & & 然 & \multirow{5}{*}{ Individu complet } & $0-6$ mois & & & & & & \\
\hline & 14 & & & NO/SE & & $\begin{array}{c}0-1 \text { an } \\
9-10 \text { mois } \\
\text { lunaires }\end{array}$ & \multirow{4}{*}{ Indet. } & $\begin{array}{l}\text { Décubitus latéral droit } \\
\text { avec les membres fléchis }\end{array}$ & \multirow{4}{*}{ Aucun } & & \multirow{4}{*}{ Non } & \\
\hline & 15 & & - & $\mathrm{N} / \mathrm{s}$ & & $2-9$ mois & & $\begin{array}{l}\text { Décubitus latéral gauche } \\
\text { avec les membres fléchis }\end{array}$ & & & & \\
\hline & 16 & \multirow{2}{*}{ Entre deux bols céramiques } & - & $\begin{array}{c}\text { Non } \\
\text { Nopervable }\end{array}$ & & 5 mois lunaires & & $\begin{array}{l}\text { Décubitus latéral droit } \\
\text { avec les membres fléchis }\end{array}$ & & & & \\
\hline & 17 & & - & & & 4 mois lunaires & & Non observable & & & & \\
\hline \multirow{6}{*}{ Tum 190} & & \multirow{6}{*}{ Ciste } & \multirow[b]{2}{*}{ oui } & \multirow{2}{*}{$\mathrm{E} / \mathrm{O}$} & Individu perturbé & $20-49$ ans & Féminin & \multirow{2}{*}{\multicolumn{2}{|c|}{ Non observable }} & \multirow{3}{*}{ Aucun } & \multirow{6}{*}{ Oui } & \multirow{6}{*}{$\begin{array}{l}\text { Dumas-Lattaque P, Courtaud P, Allard AC (2020) Area 15. In Rohmer J (ed) } \\
\text { Mission archéologique de Thaj. Rapport des campagnes 2019/2020. Non publié. }\end{array}$} \\
\hline & 1 & & & & Fragments & Immature & Indet. & & & & & \\
\hline & 2 & & \multirow[b]{2}{*}{ non } & \multirow[b]{2}{*}{$\mathrm{N}-\mathrm{NE} / \mathrm{S}-\mathrm{SO}$} & \multirow{4}{*}{ Individu perturbé } & $20-30$ ans & Masculin & \multicolumn{2}{|c|}{ Non observable } & & & \\
\hline & 3 & & & & & $>30$ ans & - & Décubitus & $\begin{array}{l}\text { Coffrage en bois? } \\
\text { Enveloppe souple? }\end{array}$ & Éléments de fer & & \\
\hline & 4 & & $\begin{array}{c}\text { oui, } \\
\text { en partie }\end{array}$ & $\mathrm{N} / \mathrm{S}$ & & $20-49$ ans & \multirow{2}{*}{ Masculin } & \multirow{2}{*}{$\begin{array}{l}\text { Décubitus, } \\
\text { membres inf. en extension }\end{array}$} & & & & \\
\hline & 5 & & \begin{tabular}{|l|}
$\begin{array}{l}\text { oui, dont } 1 \\
\text { incomplette }\end{array}$ \\
\end{tabular} & $\mathrm{NO} / \mathrm{SE}$ & & $25-30$ ans & & & Coffrage en bols? & Aucun & & \\
\hline & & & & & & Adulte & & & & & & 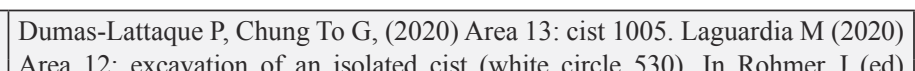 \\
\hline Ciste 1003 & 1 & Ciste & non & NE/SO & Fragments & Immature & & Non observable & & Monnale, elements en ter & Oul & 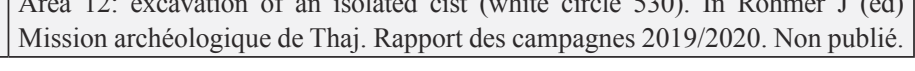 \\
\hline CB 530 & 1 & Ciste & \begin{tabular}{|l|} 
oui, dont 1 \\
incomplete
\end{tabular} & N-NE/S-SO & Fragments & Adulte & & Non observable & & $\begin{array}{l}\text { Céramique renverśé sur les } \\
\text { dalles de couverture }\end{array}$ & Oui & $\begin{array}{l}\text { Laguardia M (2020) Area 12: excavation of an isolated cist (white circle 530). } \\
\text { In Rohmer J (ed) Mission archéologique de Thaj. Rapport des campagnes } \\
\text { 2019/2020. Non publié. }\end{array}$ \\
\hline Ciste 1048 & 1 & Ciste & $\begin{array}{c}\text { oui, en } \\
\text { partie }\end{array}$ & $\mathrm{N} / \mathrm{S}$ & Aucun & & & Non observ & & & Oui & $\begin{array}{l}\text { Bibby G (1973) Preliminary Survey in East Arabia. Reports of the Danish } \\
\text { Archaeological Expedition to the Arabian Gulf, Vol 2, Jutland Archaeological } \\
\text { Society Publications 12, Gyldendal, Copenhagen, pp 25-26. } \\
\end{array}$ \\
\hline СВ 633 & 1 & Structure de pierres & & & & & & Non mentionné & & & & $\begin{array}{l}\text { Bibby G (1973) Preliminary Survey in East Arabia. Reports of the Danish } \\
\text { Archaeological Expedition to the Arabian Gulf, Vol 2, Jutland Archaeological } \\
\text { Society Publications 12, Gyldendal, Copenhagen, p2 } 26-27 \text {. } \\
\end{array}$ \\
\hline $\begin{array}{c}\text { Tum } 606 \\
\text { (Tell al-Zayir) }\end{array}$ & 1 & Ciste & oui & $\mathrm{N} / \mathrm{S}$ & Individu complet & 5-6 ans & Indet. & $\begin{array}{l}\text { Décubitus, membres sup. et inf. } \\
\text { en extension }\end{array}$ & Non mentionné & $\begin{array}{l}\text { Bijoux, appliques, masque et } \\
\text { gant en or, objets en métal } \\
\text { (pieds de lit funéraire anthropo- } \\
\text { morphique, bols etc.), perles } \\
\end{array}$ & Non & $\begin{array}{l}\text { al-Hashash A, al-Zayer WA, al-Saif ZA, al-Sanna SH, al-Hajiri MY (2001) } \\
\text { Report on the Archaeological Excavations at Thaj. Atlal 16: 23-26. }\end{array}$ \\
\hline \begin{tabular}{c|}
$\mathrm{CB} 992$ \\
(Cercle no. 1)
\end{tabular} & 1 & Ciste & $\begin{array}{c}\text { oui, en } \\
\text { partie }\end{array}$ & $\mathrm{NO} / \mathrm{SE}$ & Fragment & & & Non observable & & Aucun & Oui & $\begin{array}{l}\text { al-Hashash A, al-Saif ZA, al-Sanna SH, al-Abduljabar N (2002) Report on the } \\
\text { excavations of Thaj. Atlal 17: 17-21. }\end{array}$ \\
\hline \begin{tabular}{c|c|} 
CB 998 \\
(Cercle no. 2) \\
\end{tabular} & 1 & Ciste & $\begin{array}{c}\text { oui, en } \\
\text { partie }\end{array}$ & $\mathrm{E} / \mathrm{O}$ & Fragments & & & Non observable & & Aucun & Oui & al-Hashash A (2005) Report on the excavation of Thaj. Atlal 18: 19-21. \\
\hline & 1 & Ciste & & $\mathrm{N} / \mathrm{S}$ & & & & $\begin{array}{l}\text { Décubitus latéral gauche } \\
\text { avec les membres fléchis }\end{array}$ & & \begin{tabular}{|l|} 
Céramique renversée sur \\
les dalles de couverture. Bijoux \\
en or et pierres semi-précieuses
\end{tabular} & & \\
\hline & 2 & & & $\mathrm{O} / \mathrm{E}$ & & & & Décubitus latéral droit & & $\begin{array}{c}\text { Deux bagues en or, } \\
\text { brûle-encens cuboiide }\end{array}$ & & \\
\hline $\begin{array}{l}\text { Tum } 193 \\
\text { (Tell 1c) }\end{array}$ & 3 & & oui & $\mathrm{N} / \mathrm{S}$ & Individu complet & Non me & ionné & avec les membres fléchis & Non mentionné & & Non & al-Hashash A (2006) Brief report on the excavations at Thaj. Atlal 19:15-19. \\
\hline & 5 & Fosse rectangularre & & $\mathrm{S} / \mathrm{N}$ & & & & & & Aucun & & \\
\hline & 6 & & & NO/SF & & & & $\begin{array}{l}\text { Décubitus latéral gauche } \\
\text { avec les membres fléchis }\end{array}$ & & & & \\
\hline & 7 & & & NO/SE & & & & & & & & \\
\hline
\end{tabular}




\begin{tabular}{|c|c|c|c|c|c|c|c|c|c|c|c|c|}
\hline Tombe & Sep. & Type de sépulture & \begin{tabular}{c|} 
Dalles de \\
couverture
\end{tabular} & Orientation & Restes squelettiques & Âge (1) & Sexe (2) & Position & Contenant & Mobilier & Pillage & Référence \\
\hline \multirow{12}{*}{$\begin{array}{l}\text { Tum } 193 \\
\text { (Tell 1c) }\end{array}$} & 8 & \multirow{6}{*}{ Fosse rectangulaire } & \multirow{6}{*}{ oui } & \multicolumn{6}{|c|}{ Non observable } & \multirow{12}{*}{ Aucun } & \multirow{12}{*}{ Non } & \multirow{12}{*}{ al-Hashash A (2006) Brief report on the excavations at Thaj. Attal 19:15-19 } \\
\hline & 9 & & & \multirow{3}{*}{$\mathrm{NO} / \mathrm{SE}$} & \multirow{11}{*}{ Individu complet } & \multirow{11}{*}{\multicolumn{2}{|c|}{ Non mentionné }} & $\begin{array}{l}\text { Décubitus latéral gauche } \\
\text { avec les membres fféchis }\end{array}$ & \multirow{11}{*}{ Non mentionné } & & & \\
\hline & 10 & & & & & & & $\begin{array}{l}\text { Décubitus latéral droit } \\
\text { avec les membres fféchis }\end{array}$ & & & & \\
\hline & 11 & & & & & & & $\begin{array}{l}\text { Décubitus latéral gauche } \\
\text { avec les membres fléchis }\end{array}$ & & & & \\
\hline & 12 & & & $\mathrm{~N} / \mathrm{S}$ & & & & $\begin{array}{l}\text { Décubitus latéral droit } \\
\text { avec les membres fléchis }\end{array}$ & & & & \\
\hline & 13 & & & \multirow{2}{*}{$E / O$} & & & & $\begin{array}{l}\text { Décubitus latéral gauche } \\
\text { avec les membres fléchis }\end{array}$ & & & & \\
\hline & 14 & Fosse ovoidde en pleine terre & non & & & & & Décubitus latéral droit & & & & \\
\hline & 15 & \multirow{5}{*}{ Fosse rectangulaire } & \multirow{5}{*}{ oui } & $\mathrm{N} / \mathrm{S}$ & & & & avec les membres fléchis & & & & \\
\hline & 16 & & & $E / O$ & & & & $\begin{array}{l}\text { Décubitus latéral gauche, } \\
\text { membres inf. en extension, } \\
\text { mains derrière le cou }\end{array}$ & & & & \\
\hline & 17 & & & $\mathrm{E} / \mathrm{O}$ & & & & Décubitus latéral gauche & & & & \\
\hline & 18 & & & SO/NE & & & & avec les membres fléchis & & & & \\
\hline & 19 & & & SE/NO & & & & $\begin{array}{c}\text { Décubitus latéral droit } \\
\text { avec les membres fléchis }\end{array}$ & & & & \\
\hline
\end{tabular}

